Pure and Applied Mathematics Quarterly

Volume 6, Number 1

(Special Issue: In honor of

John Tate, Part 2 of 2$)$

$209-243,2010$

\title{
Generators and Relations for the Étale Fundamental Group
}

\author{
Max Lieblich and Martin Olsson \\ Dedicated to John Tate
}

\begin{abstract}
If $C$ is a smooth curve over an algebraically closed field $k$ of characteristic exponent $p$, then the structure of the maximal prime-to- $p$ quotient of the étale fundamental group is known by analytic methods. In this paper, we discuss the properties of the fundamental group that can be deduced by purely algebraic techniques. We describe a general reduction from an arbitrary curve to the projective line minus three points, and show what can be proven unconditionally about the maximal pro-nilpotent and pro-solvable quotients of the prime-to- $p$ fundamental group. Included is an appendix which treats the tame fundamental group from a stack-theoretic perspective.

keywords: étale fundamental group, tame fundamental group, hyperelliptic curves.
\end{abstract}

\section{INTRODUCTION}

Let $k$ be an algebraically closed field of characteristic exponent $p$. For a connected scheme $X / k$ with a geometric point $\bar{x} \rightarrow X$, let $\pi_{1}^{\prime}(X, \bar{x})$ denote the maximal prime-to- $p$ quotient of the étale fundamental group of $X$.

The motivation for this note is the following problem, posed in [7, X.2.7].

Received Mar 15, 2007. 
Problem 1.1. Let $(C, \bar{x} \rightarrow C) / k$ be a connected smooth proper pointed curve of genus $g$ over $k$. Give an algebraic proof of the fact (which follows from the comparison between the étale fundamental group and the topological fundamental group over $\mathbb{C})$ that the group $\pi_{1}^{\prime}(C, \bar{x})$ is the maximal prime-to-p quotient of the profinite completion of the free group on $2 g$ generators $\gamma_{1}, \sigma_{1}, \gamma_{2}, \sigma_{2}, \ldots, \gamma_{g}, \sigma_{g}$ modulo the relation

$$
\left[\gamma_{1}, \sigma_{1}\right]\left[\gamma_{2}, \sigma_{2}\right] \cdots\left[\gamma_{g}, \sigma_{g}\right]=1
$$

This appears to be a deep problem. For example, a solution would likely shed light on the inverse Galois problem.

There is also a version of Problem 1.1 for punctured curves.

Problem 1.2. Let $C / k$ be a smooth complete connected curve, $p_{1}, \ldots, p_{n} \in C(k)$ distinct points $(n \geq 1)$, and let $\widetilde{C}=C-\left\{p_{i}\right\}_{i=1}^{n}$. Find an algebraic proof that for any geometric point $\bar{x} \rightarrow \widetilde{C}$ the group $\pi_{1}^{\prime}(\widetilde{C}, \bar{x})$ is isomorphic to the maximal prime-to-p quotient of the profinite completion of the free group on $2 g+n-1$ generators.

It is apparently known to experts that in order to solve Problem 1.1 it suffices to solve Problem 1.2 for $\mathbb{P}^{1}-\{0,1, \infty\}$ (in particular, Fujiwara has apparently obtained many of the results of this paper using the log étale fundamental group [4]). Our aim in this paper is to write out some aspects of this bit of folklore, and to explain some properties of the fundamental group that can be proven using algebraic methods.

In order not to confuse the reader, from now on all stated results will be proven using only algebraic methods (so the content of the following theorems is that they can be proven purely algebraically). Write $F_{n}$ for the free profinite group on $n$ generators and write $S_{g}$ for the quotient of $F_{2 g}$ with generators $\gamma_{1}, \sigma_{1}, \ldots, \gamma_{g}, \sigma_{g}$ by the closed normal subgroup generated by $\left[\gamma_{1}, \sigma_{1}\right] \cdots\left[\gamma_{g}, \sigma_{g}\right]$. In what follows, the superscript $t$ indicates the tame fundamental group, while the superscript ' indicates the prime-to- $p$ quotient of the fundamental group. Similarly, given a profinite group $G$ and a class of finite groups $\mathscr{C}$ satisfying the conditions in Paragraph 2.4, we write $G^{\mathscr{C}}$ to indicate the maximal pro- $\mathscr{C}$ quotient. (The reader is referred to $\S 2$ for definitions and notation.) For example, the superscript sol (resp. nil) indicates the maximal pro-solvable (resp. maximal pro-nilpotent) quotient.

Theorem 1.3. Let the notation be as in Problem 1.1. 
(i) There is an isomorphism $\pi_{1}^{\prime}(C, \bar{x})^{\text {nil }} \simeq\left(S_{g}^{\prime}\right)^{\text {nil }}$.

(ii) Suppose the groups in $\mathscr{C}$ have orders prime to $p$. If $\pi_{1}^{\mathscr{C}}\left(\mathbb{P}^{1} \backslash\{0,1, \infty\}, \bar{x}\right)$ is topologically freely generated by a pair of ramification elements at 0 and 1 (see $\S 3)$, then the maximal pro- $\mathscr{C}$ quotient $\pi_{1}^{\mathscr{C}}(C, \bar{x})$ is isomorphic to the maximal pro- $\mathscr{C}$ quotient $F_{2 g}^{\mathscr{C}}$ modulo the closed normal subgroup generated by a non-trivial element lying in the closure of the commutator subgroup $\left[F_{2 g}^{\mathscr{C}}, F_{2 g}^{\mathscr{C}}\right]$.

Regarding Problem 1.2 we have the following.

Theorem 1.4. Let the notation be as in Problem 1.2, and let $\mathscr{C}$ be a class of finite groups of prime-to-p orders satisfying the conditions in Paragraph 2.4.

(i) If $\pi_{1}^{\mathscr{C}}\left(\mathbb{P}^{1} \backslash\{0,1, \infty\}, \bar{x}\right)$ is topologically freely generated by a pair of ramification elements at 0 and 1 , then $\pi_{1}^{\mathscr{C}}(\widetilde{C}, \bar{x})$ is isomorphic to $\left(F_{2 g+n-1}\right)^{\mathscr{C}}$.

(ii) In general $\pi_{1}^{\prime}(\widetilde{C}, \bar{x})^{\mathrm{sol}}$ is topologically finitely generated.

(iii) There is an isomorphism $\pi_{1}^{\prime}(\widetilde{C}, \bar{x})^{\text {nil }} \simeq\left(F_{2 g+n-1}^{\prime}\right)^{\text {nil }}$.

We remark that Theorem 1.4(ii) is clearly false without the prime-to-characteristic hypothesis: it is easy to see by elementary calculations in étale cohomology that the abelianization is not a free $\mathbb{Z}$-module, as its $p$-part is too big by Abhyankar's conjecture (proven by Raynaud [13] for the punctured projective line and Harbater [5] in general).

Using these results we can also prove using only algebraic techniques the following finiteness results.

Theorem 1.5. Let $X / k$ be an irreducible scheme of finite type and $\bar{x} \rightarrow X a$ geometric point. Then $\pi_{1}^{\prime}(X, \bar{x})^{\text {sol }}$ is topologically finitely generated.

Remark 1.6. A priori, it may seem that $\pi_{1}^{t}(X, \bar{x})^{\text {sol }}$ depends upon a chosen compactification of $X$. We refer the reader to Paragraphs A.2 and A.4 for basic facts about the tame fundamental group, and to Lemma A.5 for the fact that the notion is independent of the choice of compactification (and in fact does not require a regular compactification to exist).

This also gives a purely algebraic proof of an open version of the Lang-Serre theorem [7, X.2.12] for solvable groups. 
Corollary 1.7. Let $X / k$ be a scheme of finite type, and let $G$ be a finite solvable group of order prime to $p$. Then the number of tamely ramified Galois covers of $X$ with group $G$ is finite.

In X.1.10 of [7], the reader will find wildly-ramified counterexamples to Corollary 1.7 for $X=\mathbb{A}_{k}^{1}$ and $G=\mathbb{Z} / p \mathbb{Z}$ (in the form of a continuous family of Artin-Schreier coverings).

The key to all of the results in this paper is a general dévissage which enables one to deduce results for arbitrary curves from results for $\mathbb{P}^{1}-\{0,1, \infty\}$. This is described in $\S 3$.

Remark 1.8. With notation as in Problem 1.2, the main result of Borne and Emsalem in [1] gives an abstract isomorphism between the prime-to- $p$ prosolvable quotient $F_{2 g+n-1}^{\prime \text { solv }}$ and $\pi_{1}^{\prime}(\widetilde{C}, \bar{x})^{\text {solv }}$. Their method, however, does not show that the generators can be chosen to be ramification elements in the sense of $\S 3$.

Notation 1.9. If $G$ is a profinite group and if $X \subset G$ is a subset, then the closed normal subgroup of $G$ generated by $X$ is by definition the intersection of all closed normal subgroups of $G$ containing $X$.

If $g$ and $n$ are nonnegative integers, we denote by $\mathscr{M}_{g, n}$ the Artin stack of $n$-pointed proper smooth curves of genus $g$.

We write $\widehat{\mathbb{Z}}(1)$ for $\lim _{N} \boldsymbol{\mu}_{N}(\overline{\mathbb{Q}})$.

1.10. Acknowledgments. The authors are grateful to Niels Borne, David Harbater, Nick Katz, Fabrice Orgogozo, Brian Osserman, Bjorn Poonen, Akio Tamagawa, and Ravi Vakil for helpful conversations, and to Brian Conrad, Tamás Szamuely, and the referee for very careful readings of an earlier version of this paper. Lieblich was partially supported by an NSF Postdoctoral Fellowship and Olsson was partially supported by NSF grant DMS-0714086 and an Alfred P. Sloan Fellowship.

\section{2. $\mathscr{C}$-GROUPS}

2.1. It is useful to have a formalism for dealing with various quotients of $\pi_{1}$ in a uniform manner. We adopt a well-known method and include this section primarily for the sake of notation.

Let $\mathscr{C}$ be a full subcategory of the category of finite groups such that 
(1) If $G, H \in \mathscr{C}$ then $G \times H \in \mathscr{C}$.

(2) If $H \subset G$ and $G \in \mathscr{C}$ then $H \in \mathscr{C}$.

Given a profinite group $\mathfrak{G}$, there is a maximal pro- $\mathscr{C}$ quotient $\mathfrak{G} \rightarrow \mathfrak{G}^{\mathscr{C}}$ which gives a left adjoint to the natural inclusion of the category of pro- $\mathscr{C}$ groups in the category of profinite groups. In order to adhere to common conventions, given a pointed scheme $(X, *)$ we will write $\pi_{1}^{\mathscr{C}}(X, *)$ for $\pi_{1}(X, *)^{\mathscr{C}}$. The categories $\mathscr{C}$ which we will encounter most are the following.

(1) $\mathscr{C}={ }^{\prime}$, the category of finite groups of order prime to the characteristic exponent of the base field.

(2) $\mathscr{C}=$ sol, the category of solvable finite groups.

(3) $\mathscr{C}=$ nil, the category of nilpotent finite groups.

(4) $\mathscr{C}=\ell$, the category of finite $\ell$-groups for a prime number $\ell$.

Since every nilpotent group is solvable, there is a natural surjection $\mathfrak{G}^{\text {sol }} \rightarrow \mathfrak{G}^{\text {nil }}$ for any profinite group $\mathfrak{G}$.

2.2. Note also that the functor $\mathfrak{G} \mapsto \mathfrak{G}^{\mathscr{C}}$ is right exact in the sense that if

$$
\mathfrak{G}^{\prime} \rightarrow \mathfrak{G} \rightarrow \mathfrak{G}^{\prime \prime} \rightarrow 1
$$

is an exact sequence of profinite groups, then the induced sequence

$$
\mathfrak{G}^{1 \mathscr{C}} \rightarrow \mathfrak{G}^{\mathscr{C}} \rightarrow \mathfrak{G}^{\prime 1 \mathscr{C}} \rightarrow 1
$$

is also exact. This follows from the universal property of the morphism $\mathfrak{G} \rightarrow \mathfrak{G}^{\mathscr{C}}$.

2.3. As usual, given a set $X$, one can form the free pro- $\mathscr{C}$ group on $X$, denoted $F_{X}^{\mathscr{C}}$. A concrete realization is given by taking the pro- $\mathscr{C}$ completion of the ordinary free group on $X$. The cardinality of $X$ is the rank of the free group; when $X \cong\{1, \ldots, n\}$, we will write $F_{n}^{\mathscr{C}}$ for $F_{X}^{\mathscr{C}}$.

2.4. We will assume for the remainder of this paper that $\mathscr{C}$ satisfies the following conditions.

(1) If $G, H \in \mathscr{C}$ then $G \times H \in \mathscr{C}$.

(2) If $G$ is in $\mathscr{C}$ then any subgroup of $G$ and any quotient group of $G$ is in $\mathscr{C}$.

(3) $\mathscr{C}$ is closed under extensions and contains a non-trivial group.

The reader will observe that these conditions apply to all of the classes of groups listed above, with the exception of the nilpotent groups. (In general, one 
must sacrifice certain nice functorial properties in return for the strong structure theory of nilpotent groups, which we touch upon below.)

Observe also that if $\mathscr{C}$ satisfies the above conditions, then there exists a prime number $\ell$ such that $\mathscr{C}$ contains all nilpotent $\ell$-groups. Indeed (3) implies that there exists a prime number $\ell$ such that $\mathscr{C}$ contains $\mathbb{Z} / \ell \mathbb{Z}$, and then an inductive argument using (2) implies that any nilpotent $\ell$-group is also in $\mathscr{C}$.

Lemma 2.5. Suppose

$$
1 \rightarrow \mathfrak{G}^{\prime} \rightarrow \mathfrak{G} \rightarrow H \rightarrow 1
$$

is an exact sequence of profinite groups with $H$ in $\mathscr{C}$ (so in particular $H$ is finite). If $\mathfrak{G}^{\prime} \rightarrow \mathfrak{G}^{\mathscr{C}}$ is an isomorphism then $\mathfrak{G} \rightarrow \mathfrak{G}^{\mathscr{C}}$ is an isomorphism.

Proof. It suffices to show that for any open normal subgroup $W \subset \mathfrak{G}$ there exists an open normal subgroup $V$ of $\mathfrak{G}$ contained in $W$ such that $\mathfrak{G} / V$ is in $\mathscr{C}$. Since $\mathfrak{G} \rightarrow H$ is continuous, $V:=W \cap \mathfrak{G}^{\prime}$ is open and normal in $\mathfrak{G}$. Since $\mathfrak{G}^{\prime} \rightarrow \mathfrak{G}^{\prime \mathscr{C}}$ is an isomorphism, there exists an open normal subgroup $U$ of $\mathfrak{G}^{\prime}$ contained in $V$ such that the quotient $\mathfrak{G}^{\prime} / U$ is in $\mathscr{C}$. By property (2) above, $\mathfrak{G}^{\prime} / V$, being a quotient of $\mathfrak{G}^{\prime} / U$, is in $\mathscr{C}$, whereupon, by property (3), $\mathfrak{G} / V$ is in $\mathscr{C}$.

Lemma 2.6. Let

$$
1 \rightarrow \mathfrak{G}^{\prime} \rightarrow \mathfrak{G} \rightarrow H \rightarrow 1
$$

be a short exact sequence of profinite groups, with $H$ an element of $\mathscr{C}$. Then the induced sequence of pro-C्C-completions

$$
1 \rightarrow \mathfrak{G}^{\mathscr{C}} \rightarrow \mathfrak{G}^{\mathscr{C}} \rightarrow H \rightarrow 1
$$

is exact.

Proof. As mentioned in Paragraph 2.2, the exactness of the sequence

$$
\mathfrak{G}^{\mathscr{C}} \rightarrow \mathfrak{G}^{\mathscr{C}} \rightarrow H \rightarrow 1
$$

follows from the universal property of the pro- $\mathscr{C}$-completion functor. Let $K^{\prime}$ denote the kernel of the homomorphism $\mathfrak{G}^{\prime} \rightarrow \mathfrak{G}^{\mathscr{C}}$. 
The universal property of the map $\mathfrak{G}^{\prime} \rightarrow \mathfrak{G}^{\mathscr{C}}$ implies that for any element $g \in \mathfrak{G}$ there exists a unique homomorphism $\rho_{g}: \mathfrak{G}^{\mathscr{C}} \rightarrow \mathfrak{G}^{\mathscr{C}}$ such that the diagram

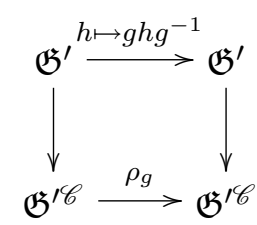

commutes. This implies that the kernel $K^{\prime}$ is also normal as a subgroup of $\mathfrak{G}$. Let $\widetilde{\mathfrak{G}}$ denote the quotient $\mathfrak{G} / K^{\prime}$ so that there is an exact sequence

$$
1 \rightarrow \mathfrak{G}^{\mathscr{C}} \rightarrow \widetilde{\mathfrak{G}} \rightarrow H \rightarrow 1 .
$$

By Lemma 2.5, the map $\widetilde{\mathfrak{G}} \rightarrow \widetilde{\mathfrak{G}}^{\mathscr{C}}$ is an isomorphism, and therefore we obtain a commutative diagram

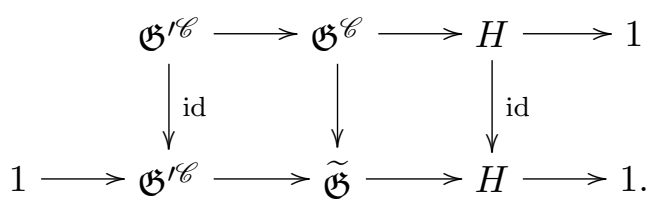

It follows that $\mathfrak{G}^{\mathscr{C}} \rightarrow \mathfrak{G}^{\mathscr{C}}$ is injective (and in fact that $\mathfrak{G}^{\mathscr{C}} \rightarrow \widetilde{\mathfrak{G}}$ is an isomorphism).

A fundamental result related to free pro- $\mathscr{C}$ groups (see Paragraph 2.3) we will use is the pro- $\mathscr{C}$ version of the Nielsen-Schreier Theorem (in a weak form). We give the statement here for the purposes of convenience and refer the reader to Theorem 5.4.4 of [17] for the full statement of the theorem and its proof (which is purely algebraic; the reader following the reference can use the results of $\S 6.1$ of [15] to provide the necessary algebraic proof of the classical version of the theorem).

Theorem 2.7 (Pro- $\mathscr{C}$ Nielsen-Schreier, weak form). If $F$ is a free pro- $\mathscr{C}$ group of finite rank $r_{F}$ and $H \subset F$ is an open subgroup of index $i$, then $H$ is a free pro- $\mathscr{C}$ group of rank $r_{H}=i\left(r_{F}-1\right)+1$.

Another useful fact is the following "Hopfian property" of profinite groups:

Theorem 2.8 ([14, 2.5.2]). Let $F$ be a topologically finitely generated profinite group and let $\varphi: F \rightarrow F$ be a surjective endomorphism. Then $\varphi$ is an isomorphism. 
2.9. Finally, for future purposes, we remind the reader of two basic facts about pro-nilpotent groups.

Lemma 2.10. Given a profinite group $\mathfrak{G}$, there is a natural topological isomorphism $\mathfrak{G}^{\text {nil }} \stackrel{\sim}{\rightarrow} \prod_{\ell} \mathfrak{G}^{\ell}$, where the right side is given the product topology.

Proof. This follows from (1) the fact that any $\ell$-group is nilpotent, and (2) the fact that any nilpotent group is naturally isomorphic to the product of its Sylow subgroups. See Prop. 2.4.3 of [17] for more details.

Lemma 2.11. Let $\mathfrak{N}$ be a pro-nilpotent group. Given generators $x_{1}^{(\ell)}, \ldots, x_{n}^{(\ell)} \in$ $\mathfrak{N}^{\ell}$ for each prime $\ell$, there are generators $x_{1}, \ldots, x_{n}$ for $\mathfrak{N}$ such that the image of $x_{i}$ in $\mathfrak{N}^{\ell}$ is $x_{i}^{(\ell)}$ for each prime $\ell$.

Proof. By Lemma 2.10, there is a natural isomorphism $\mathfrak{N} \rightarrow \prod_{\ell} \mathfrak{N}^{\ell}$. Thus, the elements $x_{i}^{(\ell)}$ give rise to elements $x_{1}, \ldots, x_{n}$ of $\mathfrak{N}$. To see that these generate $\mathfrak{N}$, it suffices to show that they generate any finite discrete nilpotent quotient of $\mathfrak{N}$. Thus, it suffices to establish the following: if $\ell_{1}, \ldots, \ell_{m}$ is a set of pairwise distinct primes and if for each $i=1, \ldots, m$ we have an $\ell_{i}$-group $G_{\ell_{i}}$ with a given choice of generators $x_{1}^{\left(\ell_{i}\right)}, \ldots, x_{n}^{\left(\ell_{i}\right)}$, then the elements $x_{j}:=\prod_{i=1}^{m} x_{j}^{\left(\ell_{i}\right)}$ generate the group $G:=G_{\ell_{1}} \times \cdots \times G_{\ell_{m}}$. This is a trivial application of the Chinese Remainder Theorem which we leave to the reader.

\section{RAMificATION MAPS}

3.1. Let $C / k$ be a connected smooth (not necessarily proper) curve, let $p \in C(k)$ be a point, and let $C^{\circ}$ denote $C-\{p\}$. Fix a geometric generic point

$$
\bar{\eta}: \operatorname{Spec}(\Omega) \rightarrow C
$$

Let $\mathscr{O}_{C, p}^{\mathrm{sh}}$ denote the strict henselization of the local ring at $p$, and let $\operatorname{Spec}\left(\mathscr{O}_{C, p}^{\mathrm{sh}}\right)^{\circ}$ denote the complement of the closed point in $\operatorname{Spec}\left(\mathscr{O}_{C, p}^{\mathrm{sh}}\right)$.

The tame fundamental group of $\operatorname{Spec}\left(\mathscr{O}_{C, p}^{\mathrm{sh}}\right)^{\circ}$ with respect to any base point is canonically isomorphic to $\widehat{\mathbb{Z}}^{\prime}(1):=\lim _{(N, \operatorname{char}(k))=1} \boldsymbol{\mu}_{N}(\overline{\mathbb{Q}})$. The map

$$
\operatorname{Spec}\left(\mathscr{O}_{C, p}^{\mathrm{sh}}\right)^{\circ} \rightarrow C^{\circ}
$$


therefore induces for every choice of dotted arrow $h$ filling in the diagram

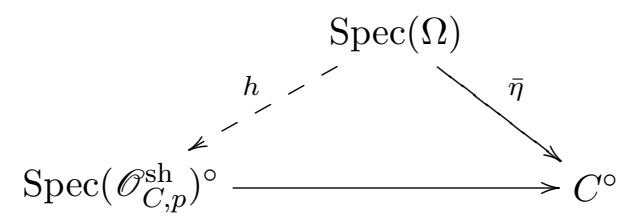

a homomorphism

$$
\widehat{\mathbb{Z}}(1) \longrightarrow \widehat{\mathbb{Z}}^{\prime}(1) \longrightarrow \pi_{1}^{t}\left(C^{\circ}, \bar{\eta}\right) .
$$

This homomorphism is well-defined up to conjugation in the following sense: if $\gamma \in \pi_{1}^{t}\left(C^{\circ}, \bar{\eta}\right)$, then the composition of (3.1.4) with conjugation by $\gamma$ is also induced by a choice of $h$ filling in (3.1.3). We denote this conjugacy class of maps by $c_{p}$. In order to specify a specific element of this conjugacy class of homomorphisms, it suffices to choose an embedding $\mathscr{O}_{C, p}^{\text {sh }} \hookrightarrow \Omega$ compatible with the given embedding $\mathscr{O}_{C, p} \hookrightarrow \Omega$. We refer to the homomorphism

$$
\rho: \widehat{\mathbb{Z}}(1) \rightarrow \pi_{1}^{t}\left(C^{\circ}, \bar{\eta}\right)
$$

obtained by making such a choice as the ramification map at $p$.

3.2. Let $K \subset \pi_{1}^{t}\left(C^{\circ}, \bar{\eta}\right)$ be the closed normal subgroup generated by the image of a ramification map $\rho$ at $p$ (see Paragraph 1.9). Note that since $K$ is by definition normal, the subgroup $K$ is independent of the choice of $\rho$ in $c_{p}$.

Proposition 3.3. The subgroup $K$ is the kernel of the surjection $\pi_{1}^{t}\left(C^{\circ}, \bar{\eta}\right) \rightarrow$ $\pi_{1}^{t}(C, \bar{\eta})$.

Proof. Let $U^{\circ} \rightarrow C^{\circ}$ be a finite étale tame cover, and let $U \rightarrow C$ be the normalization. The action of $\pi_{1}^{t}\left(C^{\circ}, \bar{\eta}\right)$ on $U_{\bar{\eta}}$ factors through $\pi_{1}^{t}(C, \bar{\eta})$ if and only if $U$ is étale over $p$. This holds if and only if the pullback

$$
U \times_{C} \operatorname{Spec}\left(\mathscr{O}_{C, p}^{\mathrm{sh}}\right) \rightarrow \operatorname{Spec}\left(\mathscr{O}_{C, p}^{\mathrm{sh}}\right)
$$

is étale. Since the formation of normalization commutes with ind-étale base change, this is true if and only if the étale cover

$$
U^{\circ} \times_{C^{\circ}} \operatorname{Spec}\left(\mathscr{O}_{C, p}^{\mathrm{sh}}\right)^{\circ} \rightarrow \operatorname{Spec}\left(\mathscr{O}_{C, p}^{\mathrm{sh}}\right)^{\circ}
$$

is trivial. This in turn is equivalent to saying that $\widehat{\mathbb{Z}}(1)$ acts trivially on $U_{\bar{\eta}}$. 
3.4. An important property of the ramification maps is that they behave well in families. Let $S=\operatorname{Spec}(V)$ be the spectrum of a discrete valuation ring with separably closed residue field, and let $s$ (resp. $\xi$ ) denote the closed (resp. generic) point of $S$. Let $C \rightarrow S$ be a proper smooth morphism of relative dimension 1 with geometrically connected fibers. Let $p_{0}, \ldots, p_{r}: S \rightarrow C$ be a collection of disjoint sections, and write $\left(C_{s}, p_{0}, \ldots, p_{r}\right)$ (resp. $\left.\left(C_{\bar{\xi}}, p_{0}, \ldots, p_{r}\right)\right)$ for the $(r+1)$ pointed curves obtained over the geometric points of $S$. Also let $C^{\circ}$ denote the complement $C-\left\{p_{0}, \ldots, p_{r}\right\}$.

Let $\bar{\eta}_{\xi}: \operatorname{Spec}\left(\Omega_{\xi}\right) \rightarrow C_{\xi}^{\circ}$ and $\bar{\eta}_{s}: \operatorname{Spec}\left(\Omega_{s}\right) \rightarrow C_{s}^{\circ}$ be geometric generic points. By Corollary A.12 we then have a surjection

$$
\pi_{1}^{t}\left(C_{\bar{\xi}}^{\circ}, \bar{\eta}_{\xi}\right) \longrightarrow \pi_{1}^{t}\left(C_{s}^{\circ}, \bar{\eta}_{s}\right)
$$

well-defined up to conjugation. For any section $p_{i}$, the conjugacy class of maps $c_{p_{i, \bar{\xi}}}$

$$
\widehat{\mathbb{Z}}(1) \rightarrow \pi_{1}^{t}\left(C_{\bar{\xi}}^{\circ}, \bar{\eta}_{\xi}\right)
$$

therefore induces a conjugacy class of maps

$$
\widehat{\mathbb{Z}}(1) \rightarrow \pi_{1}^{t}\left(C_{s}^{\circ}, \bar{\eta}_{s}\right) .
$$

Lemma 3.5. The conjugacy class of homomorphisms (3.4.1) is equal to image of the conjugacy class of maps $c_{p_{i, \bar{\xi}}}$.

Proof. This is straightforward from the definition of the specialization maps.

\section{The PUnCTURed Line}

4.1. Fix an isomorphism $\widehat{\mathbb{Z}}(1) \simeq \widehat{\mathbb{Z}}$. Let $\mathscr{C}$ be a class of groups satisfying the conditions in Paragraph 2.4 .

Let $C=\mathbb{P}_{k}^{1}$, let $\left\{p_{1}, \ldots, p_{r}\right\}$ be a finite set of points in $\mathbb{A}^{1}(k)$, and let $C^{\circ}$ denote $\mathbb{A}^{1}-\left\{p_{1}, \ldots, p_{r}\right\}$ (so $C^{\circ}$ is the complement of $r+1$ points in $\mathbb{P}^{1}$ ). Consider the following properties of $\pi_{1}\left(C^{\circ}, \bar{\eta}\right)^{\mathscr{C}}$ :

$\left(P_{1}\right)$ There exist embeddings $\mathscr{O}_{C, p_{i}}^{\mathrm{sh}} \hookrightarrow \Omega$ such that the images of the induced ramification maps

$$
\rho_{i}: \widehat{\mathbb{Z}} \simeq \widehat{\mathbb{Z}}(1) \rightarrow \pi_{1}\left(C^{\circ}, \bar{\eta}\right)^{\mathscr{C}}
$$

topologically generate $\pi_{1}\left(C^{\circ}, \bar{\eta}\right)^{\mathscr{C}}$. 
$\left(P_{2}\right)$ There exist embeddings $\mathscr{O}_{C, p_{i}}^{\mathrm{sh}} \hookrightarrow \Omega$ such that the map

$$
F_{r} \rightarrow \pi_{1}\left(C^{\circ}, \bar{\eta}\right)
$$

induced by the ramification maps induces an isomorphism on pro- $\mathscr{C}$ completions.

Let $M$ be a connected locally noetherian scheme (not necessarily a $k$-scheme), and let $p_{1}, \ldots, p_{r} \in \mathbb{A}^{1}(M)$ be a collection of sections such that for every (not necessarily closed) point $m \in M$ the fibers

$$
p_{1, m}, \ldots, p_{r, m} \in \mathbb{A}^{1}(k(m))
$$

are distinct. For each $m \in M$ we can then apply the preceding discussion to $p_{1, \bar{m}}, \ldots, p_{r, \bar{m}} \in \mathbb{A}^{1}(\overline{k(m)})$. We say that $P_{1}$ (resp. $P_{2}$ ) holds at $m$ if condition $P_{1}$ (resp. $P_{2}$ ) holds for $C_{\overline{k(m)}}$ and the points $\left\{p_{1, \bar{m}}, \ldots, p_{r, \bar{m}}\right\}$.

Lemma 4.2. (i) Let $\eta \in M$ be a point, $m \in M$ a specialization of $\eta$. If $P_{1}$ holds at $\eta$, then $P_{1}$ holds at $m$.

(ii) If the orders of the elements of $\mathscr{C}$ are invertible on $M$, and if for some $m_{0} \in M$ condition $P_{1}$ (resp. $P_{2}$ ) holds at $m_{0}$, then condition $P_{1}$ (resp. $P_{2}$ ) holds at every $m \in M$.

Proof. This follows immediately from Lemma 3.5.

Proposition 4.3. If $P_{1}$ (resp. $P_{2}$ ) holds for the set of points $\{0,1\}$ in $\mathbb{A}^{1}$, then $P_{1}$ (resp. $\left.P_{2}\right)$ holds for an arbitrary set of distinct points $\left\{p_{1}, \ldots, p_{r}\right\}$ in $\mathbb{A}^{1}$.

Proof. Since the stacks $\mathscr{M}_{0, n}$ are smooth over $\mathbb{Z}$ it suffices by Lemma 4.2 (i) to consider the case when the characteristic of $k$ is 0 (this assumption on the characteristic will be in effect until the end of this section). Furthermore, since the stacks $\mathscr{M}_{0, n}$ are connected (in fact irreducible), the validity of $P_{1}$ (resp. $P_{2}$ ) for a set $\left\{p_{1}, \ldots, p_{r}\right\}$ depends only on the number of points $r$ and not on their specific values.

It follows that if $A_{N}$ denotes $\mathbb{G}_{m}-\boldsymbol{\mu}_{N}(k)$, then it suffices to show that if $P_{1}$ (resp. $P_{2}$ ) holds for $A_{1}$, then $P_{1}$ (resp. $P_{2}$ ) also holds for $A_{N}$ for any positive integer $N$. 
Let $\pi_{N}: A_{N} \rightarrow A_{1}$ be the morphism induced by the map $\mathbb{P}^{1} \rightarrow \mathbb{P}^{1}$ sending $t$ to $t^{N}$. The map $\pi_{N}$ is a Galois finite étale cover with group $\boldsymbol{\mu}_{N}(k)$. Let

$$
\bar{\eta}: \operatorname{Spec}(\Omega) \rightarrow A_{1}
$$

be a geometric generic point, and fix a compatible collection $\left\{t^{1 / N}\right\}$ of $N$-th roots of $t$ in $\Omega$. This choice defines a lifting

$$
\bar{\eta}_{N}: \operatorname{Spec}(\Omega) \rightarrow A_{N}
$$

of $\bar{\eta}$ for every $N$. We then get a short exact sequence

$$
1 \rightarrow \pi_{1}\left(A_{N}, \bar{\eta}_{N}\right) \rightarrow \pi_{1}\left(A_{1}, \bar{\eta}\right) \rightarrow \boldsymbol{\mu}_{N}(k) \rightarrow 1,
$$

and hence by Lemma 2.6 a short exact sequence

$$
1 \rightarrow \pi_{1}\left(A_{N}, \bar{\eta}_{N}\right)^{\mathscr{C}} \rightarrow \pi_{1}\left(A_{1}, \bar{\eta}\right)^{\mathscr{C}} \rightarrow \boldsymbol{\mu}_{N}(k) \rightarrow 1 .
$$

Choose embeddings $\iota_{0}: \mathscr{O}_{\mathbb{P}^{1}, 0}^{\mathrm{sh}} \hookrightarrow \Omega$ and $\iota_{1}: \mathscr{O}_{\mathbb{P}^{1}, 1}^{\mathrm{sh}} \hookrightarrow \Omega$ defining specialization maps

$$
\rho_{0}, \rho_{1}: \widehat{\mathbb{Z}} \rightarrow \pi_{1}\left(A_{1}, \bar{\eta}\right)
$$

such that the induced map $\tau: F_{2} \rightarrow \pi_{1}\left(A_{1}, \bar{\eta}\right)$ induces a surjection (resp. isomorphism) on pro- $\mathscr{C}$-completions (by assumption this is possible). The image of $\rho_{0}(1)$ in $\boldsymbol{\mu}_{N}(k)$ is a generator of $\boldsymbol{\mu}_{N}(k)$ (the generator defining the specified isomorphism $\widehat{\mathbb{Z}} \simeq \widehat{\mathbb{Z}}(1))$. The image $\rho_{1}(1)$ of 1 in $\boldsymbol{\mu}_{N}(k)$ is the identity.

Lemma 4.4. Let $F_{2}$ be the free profinite group on generators $\gamma_{0}$ and $\gamma_{1}$, and let $\tau: F_{2} \rightarrow \pi_{1}\left(A_{1}, \bar{\eta}\right)$ be the map sending $\gamma_{0}$ to $\rho_{0}(1)$ and $\gamma_{1}$ to $\rho_{1}(1)$. Then the kernel of the composite map

$$
F_{2} \stackrel{\tau}{\longrightarrow} \pi_{1}\left(A_{1}, \bar{\eta}\right) \longrightarrow \boldsymbol{\mu}_{N}(k)
$$

is the topologically free group on the generators $\gamma_{0}^{N}$ and the elements $\gamma_{0}^{i} \gamma_{1} \gamma_{0}^{-i}$ for $0 \leq i \leq N-1$.

Proof. Let $F_{2}^{\text {disc }}$ denote the discrete free group on two generators $\gamma_{0}$ and $\gamma_{1}$ and let $a: F_{2}^{\text {disc }} \rightarrow F_{2}$ be the natural map. An elementary computation shows that the kernel $K^{\text {disc }}$ of the composite

$$
F_{2}^{\text {disc }} \rightarrow F_{2} \rightarrow \boldsymbol{\mu}_{N}(k)
$$


is generated by the elements $\gamma_{0}^{N}$ and $\gamma_{0}^{i} \gamma_{1} \gamma_{0}^{-i}$ for $0 \leq i \leq N-1$. We therefore obtain a surjection $b: F_{N+1}^{\text {disc }} \rightarrow K^{\text {disc }}$. Taking the profinite completion of the sequence

$$
F_{N+1}^{\text {disc }} \rightarrow F_{2}^{\text {disc }} \rightarrow \boldsymbol{\mu}_{N}(k) \rightarrow 1
$$

and using the exactness property in Proposition 2.2 we conclude that the map

$$
F_{N+1} \rightarrow K:=\operatorname{Ker}\left(F_{2} \rightarrow \boldsymbol{\mu}_{N}(k)\right)
$$

defined by the elements $\gamma_{0}^{N}$ and $\gamma_{0}^{i} \gamma_{1} \gamma_{0}^{-i}$ for $0 \leq i \leq N-1$ is a surjection. On the other hand, by the Nielsen-Schreier Theorem 2.7 the group $K$ is topologically free on $N+1$ generators, and therefore by the Hopfian property of profinite groups 2.8 the map $F_{N+1}^{\mathscr{C}} \rightarrow K^{\mathscr{C}}$ is an isomorphism.

We therefore obtain a commutative diagram with exact rows

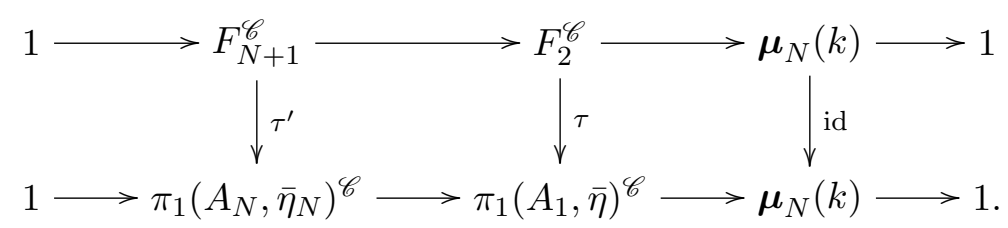

It follows that if $\tau$ is a surjection (resp. isomorphism), then $\tau^{\prime}$ is a surjection (resp. isomorphism). To complete the proof of the proposition it therefore suffices to show that the elements $\tau\left(\gamma_{0}^{N}\right)$ and $\tau\left(\gamma_{0}^{i} \gamma_{1} \gamma_{0}^{-i}\right)$ in $\pi_{1}\left(A_{N}, \bar{\eta}_{N}\right)$ are equal to the images of 1 under suitable choices of ramification maps.

The choice of $N$-th root $t^{1 / N}$ defines a morphism

$$
\iota_{0}^{N}: \operatorname{Spec}(\Omega) \rightarrow \operatorname{Spec}\left(\mathscr{O}_{\mathbb{P}^{1}, 0}^{\mathrm{sh}}\right)^{\circ}
$$

such that the induced diagram

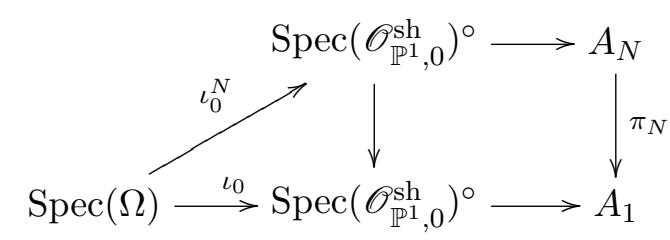

commutes. It follows that the image of 1 under the specialization map

$$
\rho_{0}^{N}: \widehat{\mathbb{Z}} \rightarrow \pi_{1}\left(A_{N}, \bar{\eta}_{N}\right)
$$

induced by $\iota_{0}^{N}$ is equal to $\tau\left(\gamma_{0}\right)^{N}$. 
Fix a lifting $\widetilde{\gamma}_{0} \in \operatorname{Gal}\left(\Omega / k\left(A_{1}\right)\right)$ of $\tau\left(\gamma_{0}\right) \in \pi_{1}\left(A_{1}, \bar{\eta}\right)$, and let $\zeta \in \boldsymbol{\mu}_{N}(k)$ be the image of 1 under the composite $\widehat{\mathbb{Z}} \rightarrow \widehat{\mathbb{Z}}(1) \rightarrow \boldsymbol{\mu}_{N}(k)$. Since the map $\mathbb{P}^{1} \rightarrow \mathbb{P}^{1}$ sending $t$ to $t^{N}$ is étale over 1 , there exist unique embeddings

$$
\iota_{\zeta^{i}}^{N}: \mathscr{O}_{\mathbb{P}^{1}, \zeta^{i}}^{\mathrm{sh}} \hookrightarrow \Omega, \quad i=0, \ldots, N-1
$$

such that the diagrams

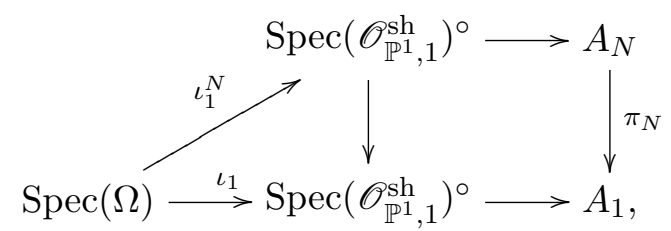

and

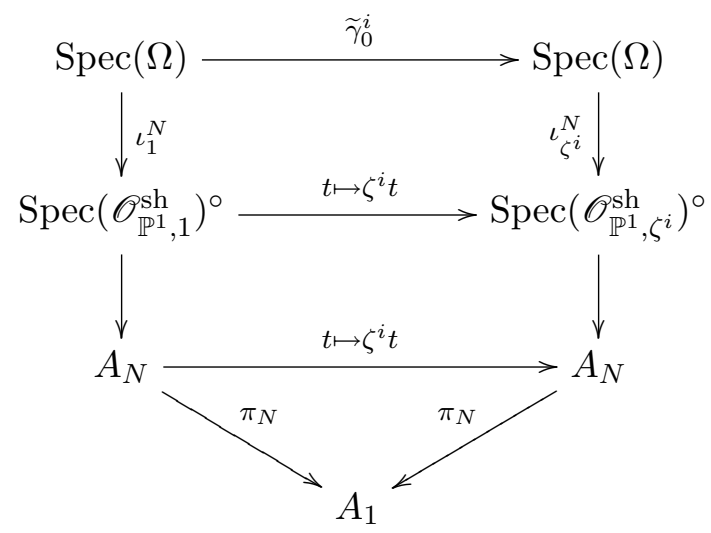

commute. If

$$
\rho_{\zeta^{i}}^{N}: \widehat{\mathbb{Z}} \rightarrow \pi_{1}\left(A_{N}, \bar{\eta}_{N}\right)
$$

denote the corresponding ramification maps, then the commutativity of (4.4.1) implies that $\rho_{1}^{N}(1)=\gamma_{1}$ and the commutativity of (4.4.2) implies that for $1 \leq$ $i \leq N-1$ we have $\rho_{\zeta^{i}}^{N}(1)=\gamma_{0}^{i} \gamma_{1} \gamma_{0}^{-i}$. The completes the proof of Proposition 4.3 .

Remark 4.5. The same argument shows that if one knows that $\pi_{1}^{\mathscr{C}}\left(A_{1}, \bar{\eta}\right)$ is finitely generated but not necessarily generated by the images of the ramification maps (where $A_{1}=\mathbb{G}_{m}-\{0,1\}$ as in the proof), then for any integer $r$ and collection of distinct points $\left\{p_{1}, \ldots, p_{r}\right\}$ in $\mathbb{A}^{1}$ the $\mathscr{C}$-completion of the fundamental group of $\mathbb{A}^{1}-\left\{p_{1}, \ldots, p_{r}\right\}$ with respect to any base point is finitely generated. Indeed, it suffices to consider the fundamental groups $\pi_{1}^{\mathscr{C}}\left(A_{N}, \bar{\eta}_{N}\right)$, and these sit 
in a short exact sequence

$$
1 \rightarrow \pi_{1}^{\mathscr{C}}\left(A_{N}, \bar{\eta}_{N}\right) \rightarrow \pi_{1}^{\mathscr{C}}\left(A_{1}, \bar{\eta}\right) \rightarrow \boldsymbol{\mu}_{N}(k) \rightarrow 1 .
$$

The finite generation of $\pi_{1}^{\mathscr{C}}\left(A_{N}, \bar{\eta}_{N}\right)$ therefore follows from the finite generation of $\pi_{1}^{\mathscr{C}}\left(A_{1}, \bar{\eta}\right)$ and the Nielsen-Schreier Theorem 2.7. Similarly, if one knows that $\pi_{1}^{\mathscr{C}}\left(A_{1}, \bar{\eta}\right)$ is topologically free then it follows that for all collections of points $\left\{p_{1}, \ldots, p_{r}\right\}$ in $\mathbb{A}^{1}(k)$ the group $\pi_{1}^{\mathscr{C}}\left(A_{N}, \bar{\eta}_{N}\right)$ is topologically free. The issue about ramification maps arises when one wants to deduce results for higher genus curves.

Remark 4.6. The assumption that the orders of the groups in $\mathscr{C}$ are invertible in $k$ can be weakened as follows. If one assumes $P_{1}$ for $\mathbb{A}^{1}-\{0,1\}$ over a field of characteristic 0 , then it follows from the argument used in the proof of Proposition 4.3 that for any field $k$ and any set of points $p_{1}, \ldots, p_{r} \in \mathbb{A}_{k}^{1}$ there exists embeddings $\mathscr{O}_{C, p_{i}}^{\mathrm{sh}} \hookrightarrow \Omega$ such that the induced maps

$$
\rho_{i}: \widehat{\mathbb{Z}} \rightarrow \pi_{1}^{t}\left(C^{\circ}, \bar{\eta}\right)^{\mathscr{C}}
$$

topologically generate $\pi_{1}^{t}\left(C^{\circ}, \bar{\eta}\right)$.

\section{Proof of Theorem 1.3(i) And Theorem 1.4(iii): Wingberg}

Let us first prove Theorem 1.3(i). Suppose $C$ is a proper smooth connected curve of genus $g$ over an algebraically closed field $k$.

Proposition 5.1 (Wingberg). There is a non-canonical isomorphism $\pi_{1}^{\ell}(C) \cong$ $S_{g}^{\ell}$ (where $S_{g}$ is defined in the paragraph preceding Theorem 1.3).

Proof. By "spreading out" and using [7, X.3.9], we may assume that $k$ has positive characteristic $p \neq \ell$. Using another spreading out argument and specialization we can further reduce to the case when $k=\overline{\mathbb{F}}_{p}$. In this case the result is due to Wingberg using the classification of Demushkin groups [18] .

Corollary 5.2. The group $\pi_{1}^{\prime}(C)^{\mathrm{nil}}$ is isomorphic to $\left(S_{g}^{\prime}\right)^{\mathrm{nil}}$.

Proof. This follows immediately from Proposition 5.1 and Lemma 2.10.

The proof of Theorem 1.4(iii) proceeds similarly, using the following substitutions: one must replace the reference to [7, X.3.9] with Corollary A.12, and the reference to [18] with [11, Chapter $\mathrm{X}, 10.1 .2]$, where the open case over the algebraic closure of a finite field is treated. 


\section{Proof of Theorem 1.4(ii)}

6.1. The main result of Borne and Emsalem [1] (building on work of Serre [16]) gives an isomorphism between $\pi_{1}^{\prime}\left(\mathbb{P}^{1} \backslash\{0,1, \infty\}\right)^{\text {sol }}$ and $F_{2}^{\prime \text { sol }}$. Given any punctured curve $C \subset \bar{C}$, choosing a generic map $\bar{C} \rightarrow \mathbb{P}^{1}$ and removing sufficiently many points from the base yields an open subcurve $U \subset C$ mapping by a finite étale morphism to an open subset $V$ of $\mathbb{P}^{1}$. By Remark 4.5 the group $\pi_{1}^{\prime}(V)^{\text {sol }}$ is topologically finitely generated, and hence $\pi_{1}^{\prime}(U)^{\text {sol }}$ is also topologically finitely generated (by the Nielsen-Schreier Theorem). Since the natural map $\pi_{1}(U) \rightarrow$ $\pi_{1}(C)$ is surjective, this completes the proof. (We have omitted the base points from this argument as we gain no traction by keeping track of them when the given data and desired conclusion are so crude.)

Remark 6.2. Note that the proof of Borne and Emsalem only gives an abstract isomorphism $\pi_{1}^{\prime}\left(\mathbb{P}^{1} \backslash\{0,1, \infty\}\right)^{\text {sol }} \cong F_{2}^{\text {ssol }}$. In particular, their methods do not show that $\pi_{1}^{\prime}\left(\mathbb{P}^{1} \backslash\{0,1, \infty\}\right)^{\text {sol }}$ is topologically generated by a pair of ramification elements, and thus we cannot apply the techniques of $\S 3$ and the following $\S 7$ to deduce results for the maximal pro-solvable prime-to- $p$ quotient which are stronger than Theorem 1.4(ii).

\section{Higher Genus CuRVes}

Next we study higher genus curves. Let $\mathscr{C}$ be a class of finite groups satisfying the assumptions in Paragraph 2.4, and assume further that for every $G \in \mathscr{C}$ the order of $G$ is invertible in $k$. Let $C / k$ be a connected proper smooth curve of genus $g$, and let $\left\{p_{0}, \ldots, p_{n}\right\}$ be a nonempty set of points of $C(k)$. Let $C^{\circ}$ denote the complement $C-\left\{p_{0}, \ldots, p_{n}\right\}$. Fix a geometric generic point

$$
\bar{\eta}: \operatorname{Spec}(\Omega) \rightarrow C^{\circ} .
$$

Consider the following properties of the $(n+1)$-pointed curve $\left(C, p_{0}, \ldots, p_{n}\right)$ :

$\left(P_{3}\right)$ There exists a homomorphism

$$
F_{2 g+n} \rightarrow \pi_{1}\left(C^{\circ}, \bar{\eta}\right)
$$

inducing a surjection on pro- $\mathscr{C}$-completions.

$\left(P_{4}\right)$ There exists a homomorphism

$$
F_{2 g+n} \rightarrow \pi_{1}\left(C^{\circ}, \bar{\eta}\right)
$$


inducing an isomorphism on pro- $\mathscr{C}$-completions.

Theorem 7.1. Suppose $P_{1}$ (resp. $\left.P_{2}\right)$ holds for $\left(\mathbb{P}^{1},\{0,1, \infty\}\right)$. Then for any $m \geq 0$ and any $(m+1)$-pointed proper smooth genus $g$ curve $\left(C,\left\{c_{0}, \ldots, c_{m}\right\}\right)$ property $P_{3}$ (resp. $\left.P_{4}\right)$ holds for $\left(C, c_{0}, \ldots, c_{m}\right)$.

Proof. As in the proof of Proposition 4.3 it suffices to consider the case when $k$ has characteristic 0 .

We assume $P_{1}$ (resp. $P_{2}$ ) for $\mathbb{P}^{1}-\{0,1, \infty\}$, so by Proposition 4.3 we have $P_{1}$ (resp. $P_{2}$ ) for any dense open in $\mathbb{P}^{1}-\{0,1, \infty\}$.

Since the moduli stack $\mathscr{M}_{g, m+1}$ classifying smooth proper curves of genus $g$ with $m+1$ marked points is irreducible (Fulton has given an algebraic proof of this fact in the appendix to [6]) and the étale fundamental group is constant in families of pointed curves, it suffices to exhibit for each pair $(g, m)$ a single $m+1$-marked genus $g$ curve for which $P_{3}$ (resp. $P_{4}$ ) holds. We do this by a careful analysis of hyperelliptic curves, which exist in every genus (as smooth members of the very ample linear system $|(g+1,2)|$ on $\mathbb{P}^{1} \times \mathbb{P}^{1}$, for example).

Let $f: C \rightarrow \mathbb{P}^{1}$ denote a hyperelliptic curve of genus $g$, fibered over the projective line with $2 g+2$ ramification points $\left\{p_{0}, \ldots, p_{2 g+1}\right\} \subset C$ and $2 g+2$ branch points $\left\{q_{0}, \ldots, q_{2 g+1}\right\} \subset \mathbb{P}^{1}$, with $p_{i}$ lying over $q_{i}$ for $i=0, \ldots, 2 g+1$. Given a positive integer $n$, choose $n$ distinct closed points $r_{1}, \ldots, r_{n} \in \mathbb{P}^{1}$ disjoint from the branch points. If $n=0$, let $\left\{r_{i}\right\}$ be the empty set. We introduce the following notations:

(1) $L_{n}=\mathbb{P}^{1} \backslash\left\{q_{0}, r_{1}, \ldots, r_{n}\right\}$

(2) $\widetilde{L}_{n}=L_{n} \backslash\left\{q_{1}, \ldots, q_{2 g+1}\right\}$

(3) $C_{n}=C \backslash\left(\left\{p_{0}\right\} \cup f^{-1}\left\{r_{1}, \ldots, r_{n}\right\}\right)$

(4) $\widetilde{C}_{n}=C_{n} \backslash\left\{p_{1}, \ldots, p_{2 g+1}\right\}$

Thus $C_{n} \rightarrow L_{n}$ is a double cover with $2 g+1$ ramification points and $\widetilde{C}_{n} \rightarrow \widetilde{L}_{n}$ is a connected $\mathbb{Z} / 2 \mathbb{Z}$-torsor. The curve $C_{n}$ is the complement of $2 n+1$ points in a hyperelliptic curve of genus $g$.

Let $\bar{\eta}: \operatorname{Spec}(\Omega) \rightarrow C^{\circ}$ be a geometric generic point, and choose maps

$$
\iota_{q_{i}}: \operatorname{Spec}(\Omega) \rightarrow \operatorname{Spec}\left(\mathscr{O}_{\mathbb{P}^{1}, q_{i}}^{\mathrm{sh}}\right)^{\circ}, \quad i=1, \ldots, 2 g+1
$$


and

$$
\iota_{r_{i}}: \operatorname{Spec}(\Omega) \rightarrow \operatorname{Spec}\left(\mathscr{O}_{\mathbb{P}^{1}, r_{i}}^{\mathrm{sh}}\right)^{\circ}, \quad i=1, \ldots, n
$$

such that the induced map

$$
\tau: F_{2 g+1+n} \rightarrow \pi_{1}\left(\widetilde{L}_{n}, \bar{\eta}\right)
$$

induces a surjection (resp. isomorphism) on pro- $\mathscr{C}$-completions. This is possible by Proposition 4.3 , since we are assuming that $P_{1}$ (resp. $P_{2}$ ) holds for $\mathbb{P}^{1}-$ $\{0,1, \infty\}$.

We then obtain a commutative diagram

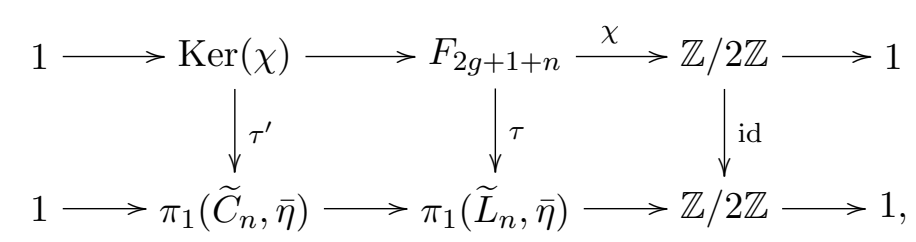

where $\chi$ is defined to be the unique homomorphism making the diagram commute.

Let $y_{i} \in F_{2 g+1+n}(i=1, \ldots, 2 g+1)$ denote the generator mapping to the image of 1 in $\pi_{1}\left(\widetilde{L}_{n}, \bar{\eta}\right)$ under the ramification map defined by $\iota_{q_{i}}$, and let $y_{j+2 g+1} \in$ $F_{2 g+1+n}(j=1, \ldots, n)$ denote the generator mapping to the image of 1 under the ramification map defined by $\iota_{r_{j}}$.

Lemma 7.2. The group $\operatorname{Ker}(\chi)$ is topologically free on the $4 g+2 n+1$ generators $g_{1 i}=y_{1} y_{i}, i=1, \ldots, 2 g+1 ; g_{j 1}=y_{j} y_{1}^{-1}, j=2, \ldots, 2 g+1 ; y_{i}, i=2 g+2, \ldots, 2 g+$ $1+n ;$ and $y_{1} y_{i} y_{1}^{-1}, i=2 g+2, \ldots, 2 g+1+n$.

Proof. By Theorem 2.7, we know that $\operatorname{Ker}(\chi)$ is topologically free of rank $4 g+$ $2 n+1$. Since $\operatorname{Ker}(\chi)$ is open and profinite completion is an exact functor, $\operatorname{Ker}(\chi)$ is the profinite completion of its intersection with the discrete free group $F_{2 g+1+n}^{\text {disc }}$ generated by the ramification elements (coming from the fixed choices of local uniformizers). By Theorem 2.8, it therefore suffices to show that the listed elements generate the discrete kernel, where we know an alternative description of the group in question: an element (word) of $F_{2 g+1+n}^{\text {disc }}$ is in $\operatorname{Ker}(\chi)$ if and only if the number of factors of the type $y_{i}^{ \pm 1}, i=1, \ldots, 2 g+1$, is even.

Let $s$ be an element of $\operatorname{Ker}(\chi) \cap F_{2 g+1+n}^{\text {disc }}$. We proceed by induction on the length $\ell(s)$ of a representation of $s$ as a product of the generators $y_{i}^{ \pm 1}$, the case $\ell(s)=0$ being trivial; thus, assume $\ell(s)>0$. By simple manipulations, each 
expression of the form $y_{i}^{ \pm 1} y_{j}^{ \pm 1}$ with $i, j \leq 2 g+1$ (and the signs in the exponents independent) is contained in the subgroup generated by the $g_{1 i}$ and $g_{j 1}$. If the first letter of $s$ has the form $y_{j}$ for $j \geq 2 g+2$, then all of the occurences of the $y_{i}$ with $i \leq 2 g+1$ take place in the remaining $\ell(s)-1$ letters, whence we are done by induction, having expressed $s$ as a product of the listed generators. Suppose that the first letter is the form $y_{i}^{ \pm 1}$ with $i \leq 2 g+1$. If the second letter has the form $y_{j}^{ \pm 1}$ with $j \leq 2 g+1$, then we are again done by induction. Thus, we may assume that the second letter has the form $y_{j}^{ \pm 1}$ with $j \geq 2 g+2$. We have that $s=y_{i}^{ \pm 1} y_{j}^{ \pm 1} s^{\prime}=\left(y_{i}^{ \pm 1} y_{1}^{-1}\right)\left(y_{1} y_{j}^{ \pm 1} y_{1}^{-1}\right)\left(y_{1} s^{\prime}\right)$ with $\ell\left(s^{\prime}\right)=\ell(s)-2$. Since the first two factors are in the subgroup generated by the listed generators and $\ell\left(y_{1} s^{\prime}\right)=\ell(s)-1$, we are done.

Lemma 7.3. The elements $g_{11}=y_{1}^{2}$ and $g_{j 1} g_{1 j}=y_{j}^{2}(j=2, \ldots, 2 g+1)$ map to the identity in $\pi_{1}\left(C_{n}, \bar{\eta}\right)$ under the composite

$$
\operatorname{Ker}(\chi) \rightarrow \pi_{1}\left(\widetilde{C}_{n}, \bar{\eta}\right) \rightarrow \pi_{1}\left(C_{n}, \bar{\eta}\right) .
$$

Moreover, the closed normal subgroup generated by their images in $\pi_{1}\left(\widetilde{C}_{n}, \bar{\eta}\right)$ is precisely the kernel of $\pi_{1}\left(\widetilde{C}_{n}, \bar{\eta}\right) \rightarrow \pi_{1}\left(C_{n}, \bar{\eta}\right)$.

Proof. For each $i=1, \ldots, 2 g+1$ choose a map

$$
\iota_{p_{i}}: \mathscr{O}_{C, p_{i}}^{\mathrm{sh}} \hookrightarrow \Omega
$$

such that the induced diagrams

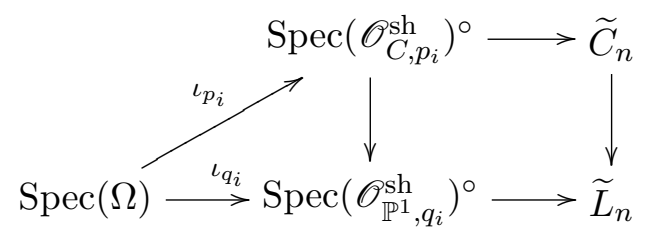

commutes.

The induced ramification map

$$
\rho_{p_{i}}: \widehat{\mathbb{Z}} \rightarrow \pi_{1}\left(\widetilde{C}_{n}, \bar{\eta}\right)
$$

then sends 1 to $g_{11}$ in the case $i=1$ and $g_{j 1} g_{1 j}$ in the case $j=2, \ldots, 2 g+2$. Since the composites

$$
\widehat{\mathbb{Z}} \stackrel{\rho_{p_{i}}}{\longrightarrow} \pi_{1}\left(\widetilde{C}_{n}, \bar{\eta}\right) \longrightarrow \pi_{1}\left(C_{n}, \bar{\eta}\right)
$$


are zero, this implies the first statement. The second follows from Proposition 3.3 .

Combining Lemmas 7.2 and 7.3 we get the following.

Corollary 7.4. The images of $g_{j 1}(j=2, \ldots, 2 g+1)$ along with $y_{i}$ and $y_{1} y_{i} y_{1}^{-1}$ $(i=2 g+2, \ldots, 2 g+n+1)$ topologically generate $\pi_{1}\left(C_{n}, \bar{\eta}\right)^{\mathscr{C}}$.

Let $H \subset \operatorname{Ker}(\chi)$ denote the closed normal subgroup generated by the elements $g_{11}$ and $g_{j 1} g_{1 j}(j=2, \ldots, 2 g+1)$, and let $Q$ denote the quotient $\operatorname{Ker}(\chi) / H$. If $\Sigma$ denotes the kernel of $\pi_{1}\left(\widetilde{C}_{n}, \bar{\eta}\right) \rightarrow \pi_{1}\left(C_{n}, \bar{\eta}\right)$, then we have a commutative diagram

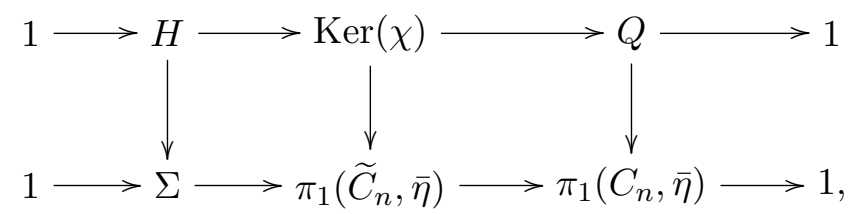

which induces a diagram of $\mathscr{C}$-completions

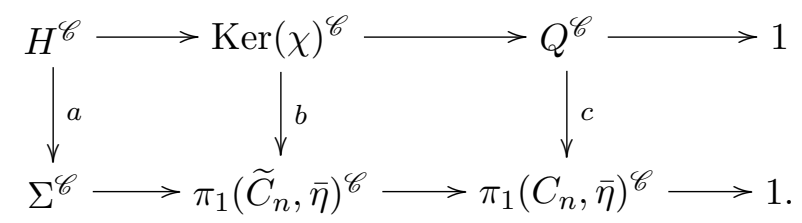

We know by Lemma 7.3 that the closed normal subgroup of $\pi_{1}\left(\widetilde{C}_{n}, \bar{\eta}\right)^{\mathscr{C}}$ generated by the image of $H^{\mathscr{C}}$ equals the image of $\Sigma^{\mathscr{C}}$. It follows that if $b$ is surjective (resp. an isomorphism) then $c$ is also surjective (resp. an isomorphism). Applying Lemma 2.6 to diagram (7.1.1) we deduce that $b$ is surjective (resp. an isomorphism), so $c$ is surjective (resp. an isomorphism). Since (by Lemma 7.2) $Q$ is equal to the profinite completion of a free group on $2 g+2 n$ generators, and $C_{n}$ is the complement of $2 n+1$ points in $C$ this proves Theorem 7.1 when $m$ is even.

To prove Theorem 7.1 for odd $m$, let $C_{n}^{\prime}$ denote the union of $C_{n}$ and the point in the preimage of $r_{1}$ corresponding to the generator $y_{2 g+2}$.

Lemma 7.5. There exists a unique point $\tilde{r}_{1} \in C(k)$ such that if

$$
h: \operatorname{Spec}\left(\mathscr{O}_{\mathbb{P}^{1}, \bar{r}_{1}}^{\mathrm{sh}}\right) \rightarrow C
$$

denotes the unique (since $C \rightarrow \mathbb{P}^{1}$ is étale over $r_{1}$ ) lifting of the map

$$
\operatorname{Spec}\left(\mathscr{O}_{\mathbb{P}^{1}, \bar{r}_{1}}^{\mathrm{sh}}\right) \rightarrow \mathbb{P}^{1}
$$


then the diagram

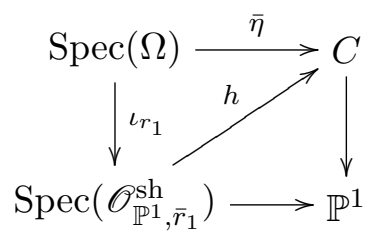

commutes. Moreover, the image in $\pi_{1}\left(C_{n}, \bar{\eta}\right)^{\mathscr{C}}$ of 1 under the ramification map

$$
\widehat{\mathbb{Z}} \rightarrow \pi_{1}\left(C_{n}, \bar{\eta}\right)
$$

defined by $h$ is equal to $y_{2 g+2}$.

Proof. The uniqueness of $\tilde{r}_{1}$ and the uniqueness of $h$ follows from the valuative criterion for separatedness applied to the morphism $C \rightarrow \mathbb{P}^{1}$. The statement that the image of 1 under the ramification map is equal to the image of $y_{2 g+2}$ follows from observing that the map

$$
\pi_{1}\left(C_{n}, \bar{\eta}\right)^{\mathscr{C}} \rightarrow \pi_{1}\left(L_{n}, \bar{\eta}\right)^{\mathscr{C}}
$$

is injective, so the ramification element is determined by its image in $\pi_{1}\left(L_{n}, \bar{\eta}\right)^{\mathscr{C}}$.

By Proposition 3.3, we have an exact sequence

$$
Z \rightarrow \pi_{1}\left(C_{n}, \bar{\eta}\right)^{\mathscr{C}} \rightarrow \pi_{1}\left(C_{n}^{\prime}, \bar{\eta}\right)^{\mathscr{C}} \rightarrow 1,
$$

where $Z$ is the closed normal subgroup generated by $y_{2 g+2}$. Let $Q^{\prime \mathscr{C}}$ denote the free pro- $\mathscr{C}$-group generated by $g_{j 1}(j=2, \ldots, 2 g+1), y_{i}(i=2 g+3, \ldots, 2 g+n+1)$, and $y_{1} y_{i} y_{1}^{-1}(i=2 g+2, \ldots, 2 g+n+1)$, and let $Q$ be as above. We then have a commutative diagram

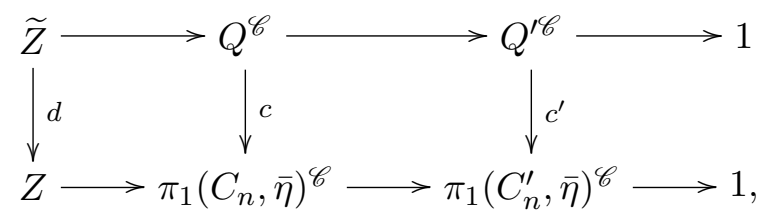

where $\widetilde{Z} \subset Q^{\mathscr{C}}$ is the closed normal subgroup generated by $y_{2 g+2}$. Since $c$ is surjective the maps $d$ and $c^{\prime}$ are surjective. It also follows that if $c$ is an isomorphism then the map $c^{\prime}$ is an isomorphism as well. 
Remark 7.6. As in Remark 4.6, the assumption that the groups in $\mathscr{C}$ have order invertible in $k$ can be weakened. If one assumes that $P_{1}$ holds for $\mathbb{P}^{1}-$ $\{0,1, \infty\}$ over a field of characteristic 0 , then it follows that for any $(n+1)$-pointed curve $\left(C, p_{0}, \ldots, p_{n}\right)$ over an arbitrary algebraically closed field $k$ there exists a homomorphism $F_{2 g+n} \rightarrow \pi_{1}\left(C^{\circ}, \bar{\eta}\right)$ inducing a surjection $F_{2 g+n} \rightarrow \pi_{1}^{t}\left(C^{\circ}, \bar{\eta}\right)^{\mathscr{C}}$.

\section{Deduction of Theorem 1.3(ii) From Theorem 1.4(i)}

8.1. Let $C / k$ be a proper smooth connected curve of genus $g$, and let $p \in C(k)$ be a point. Denote by $C^{\circ}$ the complement $C-\{p\}$, which is an affine curve. Let

$$
K \subset \pi_{1}\left(C^{\circ}, \bar{\eta}\right)
$$

be the closed normal subgroup generated by the image of a ramification map at $p$. By Proposition 3.3 the subgroup $K$ is the kernel of the surjection $\pi_{1}\left(C^{\circ}, \bar{\eta}\right) \rightarrow$ $\pi_{1}(C, \bar{\eta})$. In particular the map on maximal pro- $\mathscr{C}$ quotients

$$
\pi_{1}\left(C^{\circ}, \bar{\eta}\right)^{\mathscr{C}} \rightarrow \pi_{1}(C, \bar{\eta})^{\mathscr{C}}
$$

is surjective, with kernel generated as a closed normal subgroup by one element. Moreover, the induced map on abelianizations is an isomorphism (since it is canonically identified with the natural map on compactly supported cohomology $H_{c}^{1}\left(C^{\circ}, \widehat{\mathbb{Z}}(1)^{\mathscr{C}}\right) \rightarrow H_{c}^{1}\left(C, \widehat{\mathbb{Z}}(1)^{\mathscr{C}}\right)$, so the kernel must be generated by an element of the closure of the commutator subgroup of $\pi_{1}\left(C^{\circ}, \bar{\eta}\right)^{\mathscr{C}}$. Applying Theorem 1.4(i) gives the first part of the statement. To see that the relation is nontrivial, observe that the last statement in Paragraph 2.4 implies that there exists a prime number $\ell$ and a commutative diagram

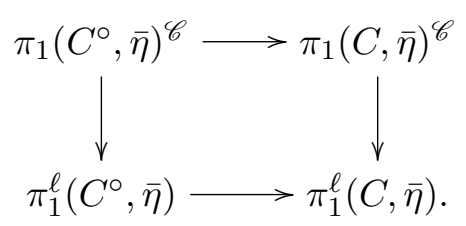

To see that the relation is nontrivial, it therefore suffices to show that it maps to a nontrivial element of $\pi_{1}^{\ell}\left(C^{\circ}, \bar{\eta}\right)$, which follows from Wingberg's Theorem 5.1.

\section{Deduction of Theorem 1.5 from Theorem 1.4(ii)}

9.1. By definition, if $f: X \rightarrow Y$ is a smooth morphism of schemes and if $D \subset X$ is a divisor, we say that $D$ has relative normal crossings if étale locally on $X$ 
there exists an étale morphism

$$
X \rightarrow Y \times \operatorname{Spec}\left(\mathbb{Z}\left[x_{1}, \ldots, x_{d}\right]\right)
$$

such that for some $s \leq d$ the divisor $D$ is equal to the inverse image of $Z\left(x_{1} \cdots x_{s}\right) \subset$ $Y \times \operatorname{Spec}\left(\mathbb{Z}\left[x_{1}, \ldots, x_{d}\right]\right)$. We say that $D$ has strict relative normal crossings if the following hold:

(i) $D$ has relative normal crossings;

(ii) There is decomposition $D=\sum_{i} D_{i}$ as a locally finite sum of effective Cartier divisors such that for every multi-index $I$ the scheme-theoretic intersection $D_{I}:=\cap_{i \in} D_{i}$ is smooth over $Y$.

Note that this implies that for every geometric point $\bar{y} \rightarrow Y$ the divisor $D_{\bar{y}} \subset$ $X_{\bar{y}}$ has strict normal crossings.

Lemma 9.2. To prove Theorem 1.5, it suffices to assume that $X$ is the complement of a strict normal crossings divisor $D$ in a smooth projective variety $\bar{X}$. More generally, the truth of Theorem 1.5 descends through arbitrary alteration (in the sense of de Jong [2]) of $X$.

Proof. Let $p: X^{\prime} \rightarrow X$ be the result of applying Chow's lemma and an appropriate alteration, so that $X^{\prime}$ is the complement of a strict normal crossings divisor in a smooth projective variety. Then by [7, IX.3.3 and IX.4.7] the morphism $p$ is of effective descent for finite étale covers. It follows from [7, IX.5.1] (and its proof) that there exists an integer $r$ and a surjection

$$
\pi_{1}\left(X^{\prime}\right)\left\langle g_{1}, \ldots, g_{r}\right\rangle \rightarrow \pi_{1}(X)
$$

from the group obtained from $\pi_{1}\left(X^{\prime}\right)$ by introducing $r$ new generators. Thus, $\pi_{1}^{\prime}(X)^{\text {sol }}$ is a quotient of

$$
\pi_{1}^{\prime}\left(X^{\prime}\right)^{\mathrm{sol}}\left\langle g_{1}, \ldots, g_{r}\right\rangle
$$

so if $\pi_{1}^{\prime}\left(X^{\prime}\right)^{\text {sol }}$ is topologically finitely generated then the group $\pi_{1}^{\prime}(X)^{\text {sol }}$ is also topologically finitely generated.

After possibly replacing $X$ by an alteration, we may assume that $X$ satisfies the conditions of Lemma 9.2.

Lemma 9.3. Let $\bar{V} \subset \bar{X}$ be a dense open subset of $\bar{X}$. Then Theorem 1.5 for $V:=\bar{V} \cap X$ implies Theorem 1.5 for $X$. 
Proof. The natural map $\pi_{1}(V) \rightarrow \pi_{1}(X)$ is surjective since $V$ is dense in the normal $X$, and therefore the induced map on prime to $p$ quotients is also surjective. It follows that if $\pi_{1}^{\prime}(V)^{\text {sol }}$ is topologically finitely generated then the same holds for $\pi_{1}^{\prime}(X)^{\text {sol }}$.

Let $D_{1}, \ldots, D_{r}$ be the irreducible components of the divisor $D$, and for a multiindex $I$ let $D_{I}$ denote $\bigcap_{i \in I} D_{i}$. By convention, if $I=\emptyset$ then $D_{I}:=\bar{X}$. Let $L$ be a very ample line bundle on $\bar{X}$. After possibly replacing $L$ by a large power of itself, we may assume that the natural map

$$
H^{0}(\bar{X}, L) \rightarrow H^{0}\left(\bar{X}, L \otimes \mathscr{O}_{D_{I}}\right)
$$

is surjective for every multi-index $I$. Let $\mathbb{P}$ denote the projective space of lines in $H^{0}(\bar{X}, L)$, and for a subscheme $Z \subset \bar{X}$ let

$$
\widehat{Z} \subset \bar{X} \times \mathbb{P}
$$

denote the incidence correspondence given by the set of (scheme-valued) points $(z, \ell)$, where $z \in Z$ and $\ell$ is a line in $H^{0}(\bar{X}, L)$ such that $x$ is contained in the vanishing locus of $\ell$. We will write $\widehat{X}$ for $\widehat{X}$. For a multi-index $I$, define $\widehat{D}_{I} \subset \widehat{X}$ to be the closed subscheme of pairs $(x, \ell)$ as above with $x \in D_{I}$.

Lemma 9.4. There exists a dense open subset $U \subset \mathbb{P}$ such that the following hold:

(i) For every multi-index $I$ (including $I=\emptyset$ ) the map $\widehat{D}_{I} \times_{\mathbb{P}} U \rightarrow U$ is smooth with geometrically connected fibers.

(ii) $\widehat{D} \times_{\mathbb{P}} U$ is a divisor in the smooth $U$-scheme $\widehat{X} \times_{\mathbb{P}} U$ with relative strict normal crossings over $U$.

Proof of 9.4. Part (i) is a consequence of Bertini's theorem and the surjectivity of the maps 9.3.1.

Part (ii) follows immediately from part (i) using the decomposition $\widehat{D} \times_{\mathbb{P}} U=$ $\sum_{i} \widehat{D}_{i} \times_{\mathbb{P}} U$

Let $M \subset \mathbb{P}$ be a line with $M \cap U$ nonempty. Since $\widehat{X} \times_{\mathbb{P}} M \rightarrow \bar{X}$ is a proper birational morphism, by Lemma 9.2 we can replace $(\bar{X}, D)$ by $\left(\widehat{X} \times_{\mathbb{P}} M, \widehat{D} \times_{\mathbb{P}} M\right)$. Applying Lemma 9.3, we can shrink the base of the pencil $M$ to $W:=U \cap M$ and therefore assume that there exists a proper smooth morphism $f: \bar{X} \rightarrow W$ 
from $\bar{X}$ to an open subset $W \subset \mathbb{P}^{1}$ such that $D$ is a divisor with relative strict normal crossings over $W$.

Let $\bar{x} \rightarrow X$ be a geometric point with image $\bar{y} \rightarrow W$. By Theorem A.15 and Paragraph 2.2 we obtain an exact sequence

$$
\pi_{1}^{\prime}\left(X_{\bar{y}}, \bar{x}\right)^{\mathrm{sol}} \rightarrow \pi_{1}^{\prime}(X, \bar{x})^{\mathrm{sol}} \rightarrow \pi_{1}^{\prime}(W, \bar{y})^{\mathrm{sol}} \rightarrow 1
$$

By induction and Theorem 1.4 it follows that if $\pi_{1}^{\prime}\left(X_{\bar{y}}, \bar{x}\right)^{\text {sol }}$ is topologically finitely generated then so is $\pi_{1}^{\prime}(X, \bar{x})^{\text {sol }}$. By induction on the dimension of $X$ we obtain Theorem 1.5.

\section{Appendix A. A STACKy approach to the tame fundamental group}

In this appendix we reprove some standard results on the tame fundamental group, used in the text, by stack-theoretic methods. The main idea is to reduce various statements about open varieties to statements about proper stacks (using Abhyankar's lemma).

A.1. For an integral separated scheme $Z$ let $\operatorname{Fet}(Z)$ denote the category of finite étale coverings of $Z$. Let $\operatorname{Fet}^{\prime}(Z)$ denote the full subcategory of $\operatorname{Fet}(Z)$ of covers whose Galois closures have degree invertible on $Z$. Both $\operatorname{Fet}^{\prime}(Z)$ and $\operatorname{Fet}(Z)$ are Galois categories. If $\bar{z} \rightarrow Z$ is a geometric point, then the inclusion $\operatorname{Fet}^{\prime}(Z) \rightarrow$ $\operatorname{Fet}(Z)$ corresponds under Galois duality to a surjective morphism of profinite groups $\pi_{1}(Z, \bar{z}) \rightarrow \pi_{1}^{\prime}(Z, \bar{z})$. If $\mathscr{C}$ denotes the category of finite groups of order invertible on $Z$, then $\pi_{1}^{\prime}(Z, \bar{z})$ is the pro- $\mathscr{C}$-completion of $\pi_{1}(Z, \bar{z})$ (in the sense of section 2).

Recall that a place of a field $\Omega$ is an equivalence class of discrete valuations on $\Omega$, where two discrete valuations $\nu_{1}, \nu_{2}: \Omega-\{0\} \rightarrow \mathbb{Z}$ are equivalent if they define the same topology on $\Omega$. A valuation is trivial if its image in $\mathbb{Z}$ is $\{0\}$, and non-trivial otherwise. The valuation $\operatorname{ring} R=\{\omega \in \Omega \mid \nu(\omega) \geq 0\}$ is a discrete valuation ring if and only if $\nu$ is non-trivial. If $Z$ is an integral separated scheme with function field $\Omega$ and $\nu$ is a place of $\Omega$ defining a valuation $\operatorname{ring} R \subset \Omega$, and if there exists a dotted arrow (necessarily unique since $Z$ is separated) filling in 
the diagram,

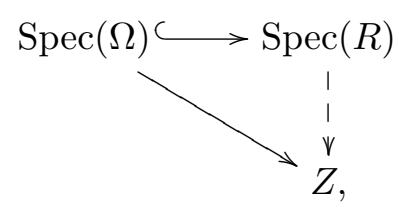

then we call the image in $Z$ of the closed point of $\operatorname{Spec}(R)$ the center of $\nu$. In this case, a valuation on $\Omega$ is trivial if and only if its center is the generic point of $Z$.

Given a set of non-trivial places $E$ of the function field of $Z$, we let $\operatorname{Fet}_{E}^{t}(Z)$ denote the full subcategory consisting of covers which are tamely ramified at the places $E$. When $E \subset Z$ is a sum of prime divisors $E=\sum E_{i}$ generically lying in the normal locus of $Z$, we will write $\operatorname{Fet}_{E}^{t}(Z)$ for $\operatorname{Fet}_{\left\{E_{i}\right\}}^{t}(Z)$. Given a basepoint $* \rightarrow Z$, the fundamental group of $\operatorname{Fet}_{E}^{t}(Z)$ at $*$ will be denoted $\left(\pi_{1}\right)_{E}^{t}(Z, *)$.

A.2. Let $V$ be a complete discrete valuation ring with separably closed residue field of characteristic $p, X / V$ a proper scheme with integral geometric fibers, and let $D \subset X$ be a divisor with relative normal crossings over $V$ (so that $X$ is smooth over $V$ in a neighborhood of $D)$. Let $\eta(\operatorname{resp} s)$ denote the generic point (resp. closed point) of $S:=\operatorname{Spec}(V)$, and let $j: X_{\eta} \hookrightarrow X$ and $i: X_{s} \hookrightarrow X$ be the inclusions. Let $X^{\circ}$ denote $X-D$. Let $E^{\text {abs }}$ denote the set of places of the function field of $X^{\circ}$ such that the induced place on the fraction field $k(\eta)$ of $V$ has a center on $\operatorname{Spec}(V)$ (the "absolute case"). Note that any such valuation on the function field of $X^{\circ}$ has a center on $X$ since $X \rightarrow \operatorname{Spec}(V)$ is proper. In what follows we write $\operatorname{Fet}^{t}\left(X^{\circ}\right)\left(\operatorname{resp} . \pi_{1}^{t}\left(X^{\circ}, \bar{x}\right)\right)$ for $\operatorname{Fet}_{E^{\text {abs }}}^{t}\left(X^{\circ}\right)\left(\operatorname{resp} .\left(\pi_{1}\right)_{E^{\text {abs }}}^{t}\left(X^{\circ}, \bar{x}\right)\right)$.

We will implicitly use the following throughout this appendix.

Lemma A.3. Given $X / V$ and $D$ as above, the inclusion $\operatorname{Fet}^{t}\left(X^{\circ}\right) \rightarrow \operatorname{Fet}_{D}^{t}\left(X^{\circ}\right)$ is an equivalence of categories, and similarly for the geometric fibers of $X$ over $V$.

Proof. It suffices to prove that any covering which is tamely ramified along $D$ must be tamely ramified at any place $\nu$ in $E^{\text {abs }}$. In what follows we fix a discrete valuation on $k(X)$ representing $\nu$, which we again denote (abusively) by $\nu$. Let $x \in X$ be the center of $\nu$. If $x \notin D$ the result is trivial, so assume $x \in D$. Completing at a closed specialization of the center point (and noting that $X$ is regular near $D$ ), we are reduced to proving the analogous statement of A.3 in the 
case where $X$ is local and regular and $D$ has strict normal crossings and hence is cut out by part of a system of parameters for $X$, say $t_{1}, \ldots, t_{s}$. Finally, since the residue field of $V$ is separably closed, we know that $V$ contains all of the prime-to- $p$ roots of unity.

Suppose $X=\operatorname{Spec} A$ and let $K$ be the function field of $X$. We need to show that if $K^{\prime} / K$ is a finite separable extension which is tamely ramified along $D$, then $K^{\prime} / K$ is also tamely ramified at $\nu$. By Abhyankar's lemma [7, XIII.3.6] there exists a positive integer $N$ relatively prime to $p$ such that the base change of $K^{\prime}$ to $B=A\left[x_{1}, \ldots, x_{s}\right] /\left(x_{1}^{N}-t_{1}, \ldots, x_{s}^{N}-t_{s}\right)$ extends to a finite étale $B$ algebra. Write $K^{\prime \prime}$ for the fraction field of $B$. Since $K^{\prime}$ extends to a finite étale $B$-algebra, to prove that $K^{\prime} / K$ is tamely ramified at $\nu$ it suffices to show that $K^{\prime \prime} / K$ is tamely ramified at $\nu$. Since the compositum of extensions which are tamely ramified at $\nu$ is again tamely ramified at $\nu$, this reduces the proof to the case when $K^{\prime}=K[x] /\left(x^{N}-t\right)$, where $N$ is an integer prime to $p$ and $t$ is a parameter defining an irreducible component of $D$. Let $R$ be the completion of the discrete valuation ring in $K$ defined by $\nu$. We then need to show that the finite extension

$$
R \rightarrow\left(R[x] /\left(x^{N}-t\right)\right)^{\sim},
$$

where the right side denotes the normalization of $R[x] /\left(x^{N}-t\right)$, has ramification indices prime to $p$.

Since $R$ contains the $N$ th roots of unity, it follows by elementary Galois theory that the integral factors of $\left(R[x] /\left(x^{N}-t\right)\right)^{\sim}$ all have the form $R[y] /\left(y^{d}-r\right)$ for some divisor $d$ of $N$ and $r$ a uniformizer in $R$. For such an extension, the ramification index must divide the degree $d$ and therefore is prime to $p$, as desired.

A.4. The discussion of Paragraph A.2 has an analogue when the base $V=\operatorname{Spec} k$ is the spectrum of a separably closed field. In particular, suppose $X / k$ is a proper geometrically integral scheme and $D \subset X$ is a strict normal crossing divisor. Write $X^{\circ}=X-D$ and let $E^{\text {abs }}$ for the set of places of the function field of $X$ for which every element of $k$ is a unit. Write $\operatorname{Fet}^{t}\left(X^{\circ}\right)$ for $\operatorname{Fet}_{E^{\text {abs }}}^{t}\left(X^{\circ}\right)$. The proof of Lemma A.3 readily yields a proof of the following.

Lemma A.5. With the preceding notation, the inclusion $\operatorname{Fet}^{t}\left(X^{\circ}\right) \rightarrow \operatorname{Fet}_{D}^{t}\left(X^{\circ}\right)$ is an equivalence of categories. 
In particular, if $Y / k$ is a geometrically integral variety with a geometric point $\bar{y} \rightarrow Y$ and $Y \hookrightarrow X_{1}$ and $Y \hookrightarrow X_{2}$ are two open immersions into proper geometrically integral $k$-varieties such that $D_{i}=X_{i}-Y, i=1,2$, is a strict normal crossings divisor, then there natural isomorphisms

$$
\left(\pi_{1}\right)_{D_{1}}^{t}\left(X_{1}, \bar{y}\right) \stackrel{\sim}{\leftarrow} \pi_{1}^{t}(Y, \bar{y}) \stackrel{\sim}{\rightarrow}\left(\pi_{1}\right)_{D_{2}}^{t}\left(X_{2}, \bar{y}\right),
$$

which shows that the computation of the tame fundamental group of $Y$ using a compactification with simple normal crossing boundary is independent of the compactification used.

A.6. The main tool in our stacky approach to the fundamental group is the socalled " $N$-th root construction." Let $Z$ be a scheme and $N \geq 1$ an integer. Let $\left(\mathscr{L}_{1}, s_{1}\right), \ldots,\left(\mathscr{L}_{r}, s_{r}\right)$ be a collection of pairs $\left(\mathscr{L}_{i}, s_{i}\right)$, where $\mathscr{L}_{i}$ is a line bundle on $Z$ and $s_{i}: \mathscr{L}_{i} \rightarrow \mathscr{O}_{Z}$ is a morphism of line bundles. Associated to this datum is a stack $\mathscr{Z}_{N} \rightarrow Z$ defined as follows (to be more precise the collection $\left\{\left(\mathscr{L}_{i}, s_{i}\right)\right\}$ should also be included in the notation, but in all cases below there should be no ambiguity as to the line bundles in question).

Consider the stack $\left[\mathbb{A}^{1} / \mathbb{G}_{m}\right]$ where $\mathbb{G}_{m}$ acts in the usual way by multiplication on $\mathbb{A}^{1}$. It is easy to see that $\left[\mathbb{A}^{1} / \mathbb{G}_{m}\right]$ is isomorphic to the stack which to any scheme $T$ associates the groupoid of pairs $(\mathscr{L}, \alpha)$, where $\mathscr{L}$ is a line bundle on $T$ and $\alpha: \mathscr{L} \rightarrow \mathscr{O}_{T}$ is a morphism of $\mathscr{O}_{T}$-modules. For any integer $N$ let

$$
p_{N}:\left[\mathbb{A}^{1} / \mathbb{G}_{m}\right] \rightarrow\left[\mathbb{A}^{1} / \mathbb{G}_{m}\right]
$$

be the map induced by the morphisms

$$
\mathbb{A}^{1} \rightarrow \mathbb{A}^{1}, \quad t \mapsto t^{N}, \quad \mathbb{G}_{m} \rightarrow \mathbb{G}_{m}, \quad u \mapsto u^{N} .
$$

By the definition for two integers $N$ and $M$ the map $p_{N M}$ factors as

$$
\left[\mathbb{A}^{1} / \mathbb{G}_{m}\right] \stackrel{p_{N}}{\longrightarrow}\left[\mathbb{A}^{1} / \mathbb{G}_{m}\right] \stackrel{p_{M}}{\longrightarrow}\left[\mathbb{A}^{1} / \mathbb{G}_{m}\right] .
$$

The maps $s_{i}: \mathscr{L}_{i} \rightarrow \mathscr{O}_{Z}$ define a morphism

$$
Z \longrightarrow\left[\mathbb{A}^{1} / \mathbb{G}_{m}\right] \times \cdots \times\left[\mathbb{A}^{1} / \mathbb{G}_{m}\right],
$$

and we define $\mathscr{Z}_{N}$ to be the fiber product of the diagram

$$
\begin{aligned}
& {\left[\mathbb{A}^{1} / \mathbb{G}_{m}\right] \times \cdots \times\left[\mathbb{A}^{1} / \mathbb{G}_{m}\right]} \\
& \downarrow p_{N} \times p_{N} \times \cdots \times p_{N} \\
& Z \longrightarrow\left[\mathbb{A}^{1} / \mathbb{G}_{m}\right] \times \cdots \times\left[\mathbb{A}^{1} / \mathbb{G}_{m}\right] \text {. }
\end{aligned}
$$


If locally we trivialize the $\mathscr{L}_{i}$ so that the maps $s_{i}$ are given by sections $t_{i} \in \mathscr{O}_{Z}$, then it follows from the definition that $\mathscr{Z}_{N}$ is the stack-theoretic quotient of

$$
\operatorname{Spec}\left(\mathscr{O}_{Z}\left[w_{1}, \ldots, w_{r}\right] /\left(w_{1}^{N}=t_{1}, \ldots, w_{r}^{N}=t_{r}\right)\right)
$$

by the action of $\boldsymbol{\mu}_{N} \times \cdots \times \boldsymbol{\mu}_{N}$ given by

$$
\left(\zeta_{1}, \ldots, \zeta_{r}\right) * w_{i}=\zeta_{i} w_{i}
$$

Note the following properties of this construction:

(i) If $N$ is invertible in $Z$ then $\mathscr{Z}_{N}$ is a Deligne-Mumford stack.

(ii) The natural map $\pi: \mathscr{Z}_{N} \rightarrow Z$ is flat and identifies $Z$ with the coarse moduli space of $\mathscr{Z}_{N}$.

(iii) Over $\mathscr{Z}_{N}$ there is a tautological collection $\left\{\left(\mathscr{M}_{i}, c_{i}\right)\right\}$ where $c_{i}: \mathscr{M}_{i} \rightarrow \mathscr{O}_{Z_{N}}$ is a morphism of line bundles on $\mathscr{Z}_{N}$ and isomorphisms $\sigma_{i}: \mathscr{M}_{i}^{N} \rightarrow \pi^{*} \mathscr{L}_{i}$ such that the diagrams

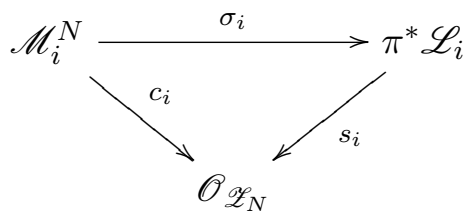

commute.

Theorem A.7. Let the notation be as in Paragraph A.2, and assume in addition that $X / V$ is smooth. Then the pullback functor

$$
j^{*}: \operatorname{Fet}^{t}\left(X^{\circ}\right) \rightarrow \operatorname{Fet}^{t}\left(X_{\bar{\eta}}^{\circ}\right)
$$

is fully faithful and the functors

$$
i^{*}: \operatorname{Fet}^{t}\left(X^{\circ}\right) \rightarrow \operatorname{Fet}^{t}\left(X_{s}^{\circ}\right)
$$

and

$$
\left.j^{*}\right|_{\operatorname{Fet}^{\prime}\left(X^{\circ}\right)}: \operatorname{Fet}^{\prime}\left(X^{\circ}\right) \rightarrow \operatorname{Fet}^{\prime}\left(X_{\bar{\eta}}^{\circ}\right)
$$

are equivalences.

Remark A.8. This theorem follows from the general theory of the log étale fundamental group $[8,4.7$ (e)]. For the convenience of the reader we give here a slightly different argument which does not (explicitly) use the theory of log geometry. 
Proof. By blowing up along an ideal supported on $D$ we can assume that $D$ is a divisor with strict normal crossings (see for example [9, 4.2.12]). Let $D_{1}, \ldots, D_{r}$ be the irreducible components of $D$, and let $\mathscr{L}_{i} \subset \mathscr{O}_{X}$ be the ideal sheaf (a line bundle) defining $D_{i}$.

For any integer $N \geq 1$ let $\mathscr{X}_{N} \rightarrow X$ denote the result of applying the $N$-th root construction applied to $X$ with the inclusions of line bundles $\left\{\mathscr{L}_{i} \subset \mathscr{O}_{X}\right\}_{i=1}^{r}$. As mentioned above, the map $\mathscr{X}_{N} \rightarrow X$ is flat, the restriction $\mathscr{X}_{N} \times_{X} X^{\circ} \rightarrow X^{\circ}$ is an isomorphism, and if $N$ is invertible in $X$ then $\mathscr{X}_{N}$ is a Deligne-Mumford stack.

For a Deligne-Mumford stack $\mathscr{Y}$ over $\operatorname{Spec}(V)$, let Fet( $\mathscr{Y})$ denote the category of finite étale morphisms of algebraic stacks $\mathscr{Y}^{\prime} \rightarrow \mathscr{Y}$, and let $\operatorname{Fet}^{\prime}(\mathscr{Y})$ denote the category of finite étale morphisms of whose Galois closures have degree invertible in $V$. (We will assume by definition that finite morphisms of stacks are representable, and not merely quasi-finite and proper.) Note that when $\mathscr{Y}$ is a scheme these categories coincide with the earlier defined categories as any Deligne-Mumford stack admitting a representable morphism to a scheme is naturally isomorphic to a scheme.

Let $\mathbb{Z}_{\geq 1}^{\prime}$ denote the set of positive integers invertible in $V$, and view $\mathbb{Z}_{\geq 1}^{\prime}$ as a category in which there exists a unique morphism $N \rightarrow M$ if $N$ divides $M$ and no morphism $N \rightarrow M$ otherwise. The $\mathscr{X}_{N}$ define a functor

$$
\mathbb{Z}_{\geq 1}^{\prime} \rightarrow(\text { stacks over } X)
$$

Remark A.9. The reader concerned about the 2-categorical nature of the right side of (A.8.1) should note that for any $N$ and $M$ a morphism $\mathscr{X}_{N} \rightarrow \mathscr{X}_{M}$, if it exists, is unique up to unique isomorphism.

Proposition A.10. The functors

$$
\begin{aligned}
& \lim _{N \in \mathbb{Z}_{\geq 1}^{\prime}} \operatorname{Fet}\left(\mathscr{X}_{N}\right) \rightarrow \operatorname{Fet}^{t}\left(X^{\circ}\right),
\end{aligned}
$$

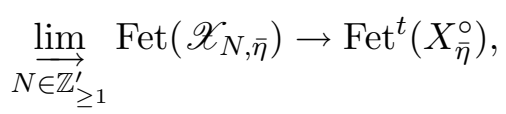

and

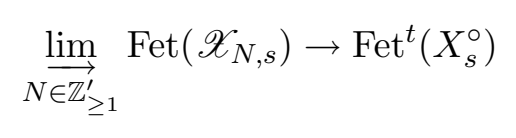


are equivalences of categories. Moreover, (A.10.1), (A.10.2), and (A.10.3) induce equivalences between the subcategories Fet'.

Proof. We prove that (A.10.1) is an equivalence of categories leaving the proof that (A.10.2) and (A.10.3) to the reader (use exactly the same argument).

For any $N \in \mathbb{Z}_{\geq 1}^{\prime}$ the open immersion $j: X^{\circ} \hookrightarrow \mathscr{X}_{N}$ is dense and $\mathscr{X}_{N}$ is normal. It follows that for any finite étale morphism $Z \rightarrow \mathscr{X}_{N}$, the stack $Z$ is equal to the normalization of $Z^{\circ}:=Z \times \mathscr{X}_{N} X^{\circ}$ in $\mathscr{X}_{N}$. This implies that (A.10.1) is fully faithful, and in addition shows that in order to prove that (A.10.1) is an equivalence we have to show the following statement: For any object $Z^{\circ} \rightarrow X^{\circ}$ of $\operatorname{Fet}^{t}\left(X^{\circ}\right)$ there exists $N \in \mathbb{Z}_{\geq 1}^{\prime}$ such that the normalization of $Z^{\circ}$ in $\mathscr{X}_{N}$ is étale over $\mathscr{X}_{N}$. This follows from the relative Abhyankar's lemma [7, XIII.3.6]. (Note that in spite of the fact that we allow covers with degrees divisible by $p$, it really does suffice to consider only covers branched to prime-to- $p$ orders to split the ramification. This is pointed out in the proof of [loc. cit.].)

From this proposition it follows that in order to prove Theorem A.7 it suffices to prove the following stack-version of $[7, \mathrm{X} .3 .9]$.

Theorem A.11. Let $\mathscr{X} \rightarrow \operatorname{Spec}(V)$ be a proper smooth Deligne-Mumford stack. Then the functor

$$
j^{*}: \operatorname{Fet}(\mathscr{X}) \rightarrow \operatorname{Fet}\left(\mathscr{X}_{\bar{\eta}}\right)
$$

is fully faithful and the functors

$$
i^{*}: \operatorname{Fet}(\mathscr{X}) \rightarrow \operatorname{Fet}\left(\mathscr{X}_{s}\right)
$$

and

$$
\left.j^{*}\right|_{\operatorname{Fet}^{\prime}(\mathscr{X})}: \operatorname{Fet}^{\prime}(\mathscr{X}) \rightarrow \operatorname{Fet}^{\prime}\left(\mathscr{X}_{\bar{\eta}}\right)
$$

are equivalences.

Proof. That $i^{*}$ is an equivalence of categories follows from the invariance of the étale site under infinitesimal thickenings and the Grothendieck existence theorem for stacks $[12,1.4]$.

That $\left.j^{*}\right|_{\mathrm{Fet}^{\prime}(\mathscr{X})}$ is an equivalence can be seen by the same technique used to prove $[7, \mathrm{X} .3 .8]$. We recall the argument. If $Z_{\eta} \rightarrow \mathscr{X}_{\eta}$ is a finite étale morphism, then it follows from the purity theorem [7, X.3.1], the "prime-to- $p$ " assumption, and the relative form of Abhyankar's lemma [loc. cit.] that there is a (totally 
ramified) finite extension $V^{\prime} \rightarrow V$ preserving the residue field such that the normalization of $\mathscr{X}_{V}$ in $\left.Z_{\eta}\right|_{V^{\prime}}$ is finite étale. Taking the special fiber yields a finite étale covering of $\mathscr{X}_{s}$, which deforms to a unique finite étale covering $Z \rightarrow \mathscr{X}_{V^{\prime}}$ (its essential preimage under $i^{*}$ ). The restriction $\left.Z\right|_{\bar{\eta}}$ is isomorphic to $\left.Z_{\eta}\right|_{\bar{\eta}}$. Thus, any element of $\operatorname{Fet}^{\prime}\left(\mathscr{X}_{\bar{\eta}}\right)$ arising by pullback from an element $\operatorname{of}^{\operatorname{Fet}^{\prime}}\left(\mathscr{X}_{\eta}\right)$ is in the essential image of $\left.j^{*}\right|_{\mathrm{Fet}^{\prime}(\mathscr{X})}$. Since $i^{*}$ is an isomorphism and remains so upon any base change $V^{\prime} \rightarrow V$, it follows that $j^{*}$ is essentially surjective. That $j^{*}$ (and hence $\left.j^{*}\right|_{\text {Fet }^{\prime}(\mathscr{X})}$ ) is fully faithful follows from a similar argument applied to (spaces of) morphisms between finite étale coverings of $\mathscr{X}$.

Corollary A.12. If $y_{\eta} \in X_{\eta}^{\circ}$ is a point specializing to $y_{s} \in X_{s}^{\circ}$, then there is a canonical surjection

$$
\pi_{1}^{t}\left(X_{\bar{\eta}}^{\circ}, \bar{y}_{\eta}\right) \rightarrow \pi_{1}^{t}\left(X_{s}^{\circ}, \bar{y}_{s}\right)
$$

of tame fundamental groups and a canonical isomorphism

$$
\pi_{1}^{\prime}\left(X_{\bar{\eta}}^{\circ}, \bar{y}_{\eta}\right) \simeq \pi_{1}^{\prime}\left(X_{s}^{\circ}, \bar{y}_{s}\right)
$$

of prime-to-p fundamental groups.

Corollary A.13. Let the notation and assumptions be as in Theorem A.7. The canonical map of topological groups

$$
\pi_{1}^{t}\left(X^{\circ}, \bar{x}\right) \rightarrow \lim _{N \in \mathbb{Z}^{\prime}} \pi_{1}\left(\mathscr{X}_{N}, \bar{x}\right)
$$

is an isomorphism, where the right side is given the inverse limit of the profinite topologies. In particular, the inverse limit topology on the right side coincides with the profinite topology.

Proof. For $N \in \mathbb{Z}^{\prime}$ let $\rho_{N}: \pi_{1}^{t}\left(X^{\circ}, \bar{x}\right) \rightarrow \pi_{1}\left(\mathscr{X}_{N}, \bar{x}\right)$ denote the surjective projection, and let $K_{N} \subset \pi_{1}^{t}\left(X^{\circ}, \bar{x}\right)$ denote the kernel. The fact that the functor

$$
\underset{N \in \mathbb{Z}^{\prime}}{\lim _{N}} \operatorname{Fet}\left(\mathscr{X}_{N}\right) \rightarrow \operatorname{Fet}^{t}\left(X^{\circ}\right)
$$

is an equivalence by Proposition A.10 implies that $\cap_{N \in \mathbb{Z}^{\prime}} K_{N}=\{1\}$. It follows that the map (A.13.1) is injective. Now the inverse limit topology makes the codomain group Hausdorff, and the termwise surjectivity makes the map in question have dense image (with respect to the inverse limit topology). It follows that the map is a closed bijection and therefore a topological isomorphism. 
Remark A.14. The same argument proving Corollary A.13 shows that if $k$ is an algebraically closed field and $X / k$ is a proper smooth scheme with a divisor $D \subset X$ with simple normal crossings, then for any geometric point $\bar{x} \rightarrow X^{\circ}:=$ $X-D$ the natural map of topological groups

$$
\pi_{1}^{t}\left(X^{\circ}, \bar{x}\right) \rightarrow \varliminf_{N \in \mathbb{Z}^{\prime}} \pi_{1}\left(\mathscr{X}_{N}, \bar{x}\right)
$$

is an isomorphism, where $\mathscr{X}_{N}$ denotes the result of applying the $N$-th root construction using the line bundles defining the irreducible components of $D$.

Theorem A.15. Let $f: X \rightarrow Y$ be a proper smooth morphism between integral Noetherian schemes with $f_{*} \mathscr{O}_{X}=\mathscr{O}_{Y}$. Let $D \subset X$ be a divisor with relative normal crossings relative to $Y$, and let $X^{\circ}=X \backslash D$. Choose a geometric point $\bar{x} \rightarrow X^{\circ}$, with image $\bar{y} \rightarrow Y$. Let $D_{X_{\bar{y}}}:=D \times_{Y} X_{\bar{y}}$ be the fiber of $D$ Then there is an exact sequence

$$
\left(\pi_{1}\right)_{D_{X_{\bar{y}}}}^{t}\left(X_{\bar{y}}^{\circ}, \bar{x}\right) \rightarrow\left(\pi_{1}\right)_{D}^{t}\left(X^{\circ}, \bar{x}\right) \rightarrow \pi_{1}(Y, \bar{y}) \rightarrow 1 .
$$

Remark A.16. Just as in $\S 1.6$ of [7], one deduces the more general exact sequence in low-degree homotopy groups (continuing to the right with $\pi_{0}$ ) when the Stein factorization of $f$ is non-trivial. When $X$ and $Y$ are proper smooth $k$-varieties, the same sequence holds with the divisor subscripts omitted.

Proof of A.15. Recall that a morphism of stacks $f: \mathscr{X} \rightarrow \mathscr{Y}$ is separable if $f$ is flat, and if for every field valued point $\operatorname{Spec}(K) \rightarrow \mathscr{Y}$ the fiber product $\mathscr{X} \times \mathscr{Y} \operatorname{Spec}(K)$ is reduced.

Lemma A.17. For every $N \in \mathbb{Z}^{\prime}$, the map $\mathscr{X}_{N} \rightarrow Y$ is separable.

Proof. Since the map $\mathscr{X}_{N} \rightarrow X$ is flat the composite $\mathscr{X}_{N} \rightarrow X \rightarrow Y$ is also flat. To verify the separability, it therefore suffices to show that for any fieldvalued point $y: \operatorname{Spec}(K) \rightarrow Y$ the fiber product $\mathscr{X}_{N} \times_{Y} \operatorname{Spec}(K)$ is reduced. This is clear because this fiber is isomorphic to the result of applying the $N$ th root construction to the divisor with simple normal crossings $D_{y} \subset X_{y}:=$ $X \times_{Y} \operatorname{Spec}(K)$.

Proposition A.18. Let $\mathfrak{f}: \mathscr{X} \rightarrow \mathscr{Y}$ be a proper separable morphism of integral Noetherian Deligne-Mumford stacks with $\mathfrak{f}_{*} \mathscr{O}_{\mathscr{X}}=\mathscr{O}_{\mathscr{Y}}$. Let $\bar{x} \rightarrow \mathscr{X}$ be a geometric point with image $\bar{y} \rightarrow \mathscr{Y}$. Then there is an exact sequence

$$
\pi_{1}\left(\mathscr{X}_{\bar{y}}, \bar{x}\right) \rightarrow \pi_{1}(\mathscr{X}, \bar{x}) \rightarrow \pi_{1}(\mathscr{Y}, \bar{y}) \rightarrow 1 .
$$


Proof. The proof is formally identical to that of Théorème X.1.3 and Corollaire X.1.4 of [7], to which we refer the reader for the details.

We use this Proposition to prove Theorem A.15 as follows. For every $N \in \mathbb{Z}^{\prime}$ we obtain an exact sequence

$$
\left(S_{N}\right): \quad \pi_{1}\left(\mathscr{X}_{N, \bar{y}}, \bar{x}\right) \rightarrow \pi_{1}\left(\mathscr{X}_{N}, \bar{x}\right) \rightarrow \pi_{1}(Y, \bar{y}) \rightarrow 1 .
$$

These sequences form a filtering inverse system of exact sequences of profinite groups: if $N \mid M$ then there is a natural morphism of exact sequences $\left(S_{M}\right) \rightarrow$ $\left(S_{N}\right)$.

The following Lemma now concludes the proof of Theorem A.15.

Lemma A.19. The induced sequence

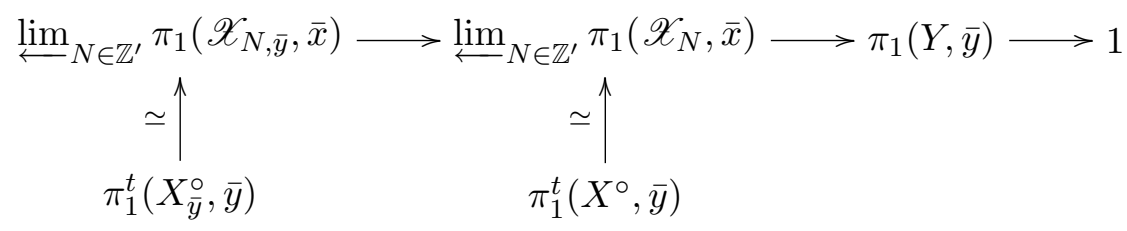

is exact.

Proof. Let $I \subset \lim _{N \in \mathbb{Z}^{\prime}} \pi_{1}\left(\mathscr{X}_{N}, \bar{x}\right)$ be the image of $\lim _{N \in \mathbb{Z}^{\prime}} \pi_{1}\left(\mathscr{X}_{N, \bar{y}}, \bar{x}\right)$. This is a closed subgroup of $\lim _{N \in \mathbb{Z}^{\prime}} \pi_{1}\left(\mathscr{X}_{N}, \bar{x}\right)$ by Corollary A.14, so it suffices to show that $I$ is dense in the kernel of the map to $\pi_{1}(Y, \bar{y})$, which is immediate.

\section{REFERENCES}

[1] N. Borne and M. Emsalem, Note sur la détermination algébrique du groupe fondamental prorésoluble d'une courbe affine, preprint http://www.arxiv.org/pdf/0708.0093.

[2] A. J. de Jong, Smoothness, semi-stability and alterations, Inst. Hautes Études Sci. Publ. Math. 83 (1996), 51-93.

[3] M. Fried and M. Jarden, Field arithmetic, Ergebnisse der Mathematik und ihrer Grenzgebiete. 3. Folge, 11, Springer-Verlag, Berlin, 1986.

[4] K. Fujiwara, Etale topology and the philosophy of log, Algebraic Geometry Symposium (Kinosaki) (1990), 116-123 (in Japanese).

[5] D. Harbater, Abhyankar's conjeture on Galois groups over curves, Invent. Math. 117 (1994), $1-25$.

[6] J. Harris and D. Mumford, On the Kodaira dimension of the moduli space of curves (with an appendix by William Fulton), Invent. Math. 67 (1982), no. 1, 23-88.

[7] A. Grothendieck, Revêtements Étales et Groupe Fondamental, Lecture Notes in Mathematics 224, Springer-Verlag, Berlin (1971). 
[8] L. Illusie, An overview of the work of K. Fujiwara, K. Kato, and C. Nakayama on logarithmic étale cohomology, Astérisque 279 (2002), 271-322.

[9] K. Kato and T. Saito, On the conductor formula of Bloch, Inst. Hautes Études Sci. Publ. Math. 100 (2004), 5-151.

[10] J. S. Milne, Étale Cohomology, Princeton University Press, Princeton, NJ (1980).

[11] J. Neukirch, A. Schmidt, and K. Wingberg, Cohomology of number fields, Grundlehren der Mathematischen Wissenschaften 323, Springer-Verlag (2000).

[12] M. Olsson, On proper coverings of Artin stacks, Advances in Mathematics 198 (2005), 93-106

[13] M. Raynaud, Rêvetements de la droite affine in caractéristique $p>0$, Invent. Math. 116 (1994), 425-462.

[14] L. Ribes and P. Zalesskii, Profinite groups, Ergebnisse der Mathematik und ihrer Grenzgebiete. 3. Folge. A Series of Modern Surveys in Mathematics, volume 40, Springer-Verlag, Berlin, 2000.

[15] D. Robinson, A Course in the Theory of Groups, Graduate Texts in Mathematics 80, Springer-Verlag, New York (1982).

[16] J.-P. Serre, Construction de revêtements étales de la droite affine en caractéristique $p$, C.R. Acad. Sci. Paris Sér I Math. 311 (1990), 341-346.

[17] J. S. Wilson, Profinite groups, Oxford University Press (1998).

[18] K. Wingberg, Ein Analogon zur Fundamentalgruppe einer Riemannschen Fläche im Zahlkörperfall, Invent. Math. 77 (1984), 557-584.

Max Lieblich

Fine Hall, Washington Road

Princeton NJ 08544-1000

Martin Olsson

Dept. of Mathematics

University of California

Berkeley, CA 94720-3840

E-mail: molsson@Math.Berkeley.EDU 